TRANSACTIONS OF THE

AMERICAN MATHEMATICAL SOCIETY

Volume 355, Number 7, Pages 2949-2971

S 0002-9947(03)03269-0

Article electronically published on March 19, 2003

\title{
THERMODYNAMIC FORMALISM FOR COUNTABLE TO ONE MARKOV SYSTEMS
}

\author{
MICHIKO YURI
}

\begin{abstract}
For countable to one transitive Markov systems we establish thermodynamic formalism for non-Hölder potentials in nonhyperbolic situations. We present a new method for the construction of conformal measures that satisfy the weak Gibbs property for potentials of weak bounded variation and show the existence of equilibrium states equivalent to the weak Gibbs measures. We see that certain periodic orbits cause a phase transition, non-Gibbsianness and force the decay of correlations to be slow. We apply our results to higherdimensional maps with indifferent periodic points.
\end{abstract}

\section{$\S 0$. INTRODUCTION}

Thermodynamic formalism for hyperbolic systems was satisfactorily established with Bowen's program ([2]). The existence of generating finite Markov partitions and analysis of Ruelle-Perron-Frobenius operators associated to Hölder potentials allow one to show the existence of unique equilibrium states that satisfy the Gibbs property (in the sense of Bowen) and exponential decay of correlations. Also, the pressure functions are analytic and there is no possibility of phase transition (nonuniqueness of equilibrium states). Furthermore, the analyticity problem is strongly related to multifractal problems and the zero of Bowen's equation determines the Hausdorff dimension of limit sets arising from certain iterated functional systems (4], [8], [12], 22]). On the other hand, phase transition, failure of the Gibbs property and slow decay of correlations can be observed for many complex systems which exhibit common phenomena in transition to turbulence (the so-called Intermittency). In this paper we shall construct mathematical models which exhibit such phenomena and for this purpose we shall establish thermodynamic formalism for non-Hölder potentials in nonhyperbolic situations in the following sense: generating Markov partitions are countable partitions and dynamical instability is subexponential (subexponential decay of cylinder sizes). More specifically, for countable to one transitive Markov systems we shall construct conformal measures $\nu$ that are weak Gibbs measures for potentials $\phi$ of weak bounded variation (WBV)(see definitions in $\S 1$ ) and show the existence of equilibrium states $\mu$ for $\phi$ equivalent to the weak Gibbs measures $\nu$. The conformal measures $\nu$ associated to $\phi$ play important roles as reference measures from the physical point of view, and absolute continuity of equilibrium states allows one to describe statistical properties of observable phenomena in the physical sense. In order to clarify typical reasons for

Received by the editors March 28, 2002 and, in revised form, September 10, 2002.

2000 Mathematics Subject Classification. Primary 28D99, 28D20, 58C40, 58E30, 37A40, 37A30, 37C30, 37D35, 37F10, 37A45. 
phase transition, non-Gibbsianness and slow decay of correlations, we introduce in $\S 4$ a notion of indifferent periodic point associated to potentials $\phi$ of WBV. Those periodic points cause failure of summable variation for potentials $\phi$ and failure of bounded distortion of local Jacobians with respect to the weak Gibbs measure $\nu$ for $\phi$ (Proposition 4). Then a construction of weak Gibbs measures $\nu$ for $\phi$ admitting indifferent periodic points implies "subexponential instability" in terms of cylinder measures (Proposition 3). Furthermore, a construction of equilibrium states $\mu$ for such $\phi$ equivalent to $\nu$ (Theorem 6, Lemma 15) allows us to show both phase transition (Corollary 2, Theorem 8) and non-Gibbsianness of equilibrium states (Theorem 5). In particular, our results are applicable to the following piecewise $C^{1}$-smooth countable to one Markov maps $T$ defined on bounded regions $X \subset \mathbb{R}^{d}$ with indifferent periodic points $\left(T^{q} x_{0}=x_{0},\left|\operatorname{det} D T^{q}\left(x_{0}\right)\right|=1\right)$ for which the potentials $-\log |\operatorname{det} D T|$ satisfy neither summable variation nor bounded distortion, so that previous results cannot be applicable.

Example A. (Inhomogeneous Diophantine approximations [13, [15, 16], 17], [19], [20], [21]). Let $X=\left\{(x, y) \in \mathbb{R}^{2}: 0 \leq y \leq 1,-y \leq x<-y+1\right\}$ and define $T: X \rightarrow X$ by

$$
T(x, y)=\left(\frac{1}{x}-\left[\frac{1-y}{x}\right]+\left[-\frac{y}{x}\right],-\left[-\frac{y}{x}\right]-\frac{y}{x}\right),
$$

where $[x]=\max \{n \in \mathbb{Z} \mid n \leq x\}(x \in \mathbb{N})$ and $[x]=\max \{n \in \mathbb{Z} \mid n<x\}(x \in$ $\mathbb{Z} \backslash \mathbb{N})$. This map admits indifferent periodic points $(1,0)$ and $(-1,1)$ with period 2, i.e., $\left|\operatorname{det} D T^{2}(1,0)\right|=\left|\operatorname{det} D T^{2}(-1,1)\right|=1$ and is related to a Diophantine approximation problem of inhomogeneous linear class.

Example B (A complex continued fraction [5], [12], [15], [22]). We can define a complex continued fraction transformation $T: X \rightarrow X$ on the diamond-shaped region $X=\left\{z=x_{1} \alpha+x_{2} \bar{\alpha}:-1 / 2 \leq x_{1}, x_{2} \leq 1 / 2\right\}$, where $\alpha=1+i$, by $T(z)=1 / z-[1 / z]_{1}$. Here $[z]_{1}$ denotes $\left[x_{1}+1 / 2\right] \alpha+\left[x_{2}+1 / 2\right] \bar{\alpha}$, where $z$ is written in the form $z=x_{1} \alpha+x_{2} \bar{\alpha},[x]=\max \{n \in \mathbb{Z} \mid n \leq x\}(x \in \mathbb{N})$ and $[x]=\max \{n \in \mathbb{Z} \mid n<x\}(x \in \mathbb{Z}-\mathbb{N})$. This transformation has an indifferent periodic orbit $\{1,-1\}$ of period 2 and two indifferent fixed points at $i$ and $-i$.

We recall previous works related to thermodynamic formalism for countable to one Markov systems. For countable Markov shifts, O. Sarig proved the existence of conformal measures and equilibrium states associated to locally Hölder potentials defined in [10] and D. Fiebig, U. Fiebig and the author proved the existence of equilibrium states for potentials satisfying bounded distortion $\left(\sup _{n \geq 1} C_{n}<\infty\right.$ in the definition of WBV) in [7. Our main Theorems 4-8 do not satisfy these assumptions and Examples A, B show that they cannot be treated by methods in [10] and in [7. Furthermore, for higher-dimensional systems that are not symbolic systems we may have crucial difficulties in verifying the positive recurrence condition imposed on potentials in both [10] and [7]. The infinite iterated functional systems that Mauldin and Urbanski studied in [8] correspond to the local inverses of piecewise conformal countable Bernoulli systems in our sense, and the method used in [8] severely relies on the Bernoulli property which fails to hold for Example B. Moreover, a Hölder-type condition, imposed on potentials for the existence of conformal measures and for establishing a variational principle, is not satisfied by the important potentials $-\log |\operatorname{det} D T|$ for both Examples A and B. 
In order to prove our theorems, we first give in $\S 2$ an appropriate definition of topological pressure for countable to one transitive Markov systems with finite range structure. Our definition coincides with the standard one by using periodic points under certain conditions (Lemma 6) which can be easily verified for higher-dimensional examples in $\S 8$. We also associate the topological pressure to the spectral radius of the Perron-Frobenius-Ruelle operator (Theorem 3). The essential issue for constructing both weak Gibbs measures $\nu$ for $\phi$ and equilibrium states $\mu$ for $\phi$ equivalent to $\nu$ is to derive Schweiger's jump transformations $T^{*}$ over full cylinders (see the definition in $\S 1$ ) with respect to which a local exponential instability (05) and a local bounded distortion (06) for potentials $\phi$ are satisfied. Then showing the existence of a zero of a generalized Bowen's equation (GBE) for derived potentials $\phi^{*}$ associated to $T^{*}$ (Lemma 7) allows one to show the existence of conformal measures that are weak Gibbs measures for $\phi$ (Theorem 4). Under a mild condition which cannot be covered by previous works, we show the existence of a zero of (GBE) in $\S 3$ by using a product formula of zeta functions (Proposition 1 ), which shows a nice relation between zeta functions for the original systems and zeta functions for the jump transformations. We also construct $\sigma$-finite conformal measures via induced maps $T_{A}$ over a single full cylinder $A$ (Theorem 7 ) in $\S 6$ by using some idea that appeared in a previous work by M. Denker and the author [5] in which no evidence of the existence of weak Gibbs conformal measures was given. We establish the existence of equilibrium states $\mu$ for $\phi$ of WBV equivalent to the weak Gibbs measure $\nu$ for $\phi$ via a jump transformation (Theorem 6 ) in $\S 5$ and via induced maps (Lemma 15) in $\S 6$. Then we can immediately see that the appearance of indifferent periodic orbits associated to $\phi$ implies a phase transition, i.e., failure of the uniqueness of equilibrium states (Corollary 2, Theorem 8). We should remark that our construction via induced maps shows the existence of (countably many) mutually singular equilibrum states. In $\S 8$ we apply our results to higher-dimensional piecewise $C^{1}$ Markov maps with indifferent periodic points. All proofs of results in $\S \S 2-3$ are postponed to the Appendix.

\section{$\S 1$. Preliminaries}

Let $(X, d)$ be a compact metric space and let $T: X \rightarrow X$ be a noninvertible map that is not necessarily continuous. Suppose that there exists a countable disjoint partition $Q=\left\{X_{i}\right\}_{i \in I}$ of $X$ such that $\bigcup_{i \in I} i n t X_{i}$ is dense in $X$ and the following properties are satisfied.

(01) For each $i \in I$ with $\operatorname{int} X_{i} \neq \emptyset,\left.T\right|_{\text {int } X_{i}}: \operatorname{int} X_{i} \rightarrow T\left(\right.$ int $\left.X_{i}\right)$ is a homeomorphism and $\left(\left.T\right|_{\text {int } X_{i}}\right)^{-1}$ extends to a homeomorphism $v_{i}$ on $\operatorname{cl}\left(T\left(\right.\right.$ int $\left.\left.X_{i}\right)\right)$.

(02) $T\left(\bigcup_{\text {int } X_{i}=\emptyset} X_{i}\right) \subset \bigcup_{\text {int } X_{i}=\emptyset} X_{i}$.

(03) $\left\{X_{i}\right\}_{i \in I}$ generates $\mathcal{F}$, the $\sigma$-algebra of Borel subsets of $X$.

We say that the triple $\left(T, X, Q=\left\{X_{i}\right\}_{i \in I}\right)$ is a piecewise $C^{0}$-invertible system. By (01), $\left.T\right|_{\text {int } X_{i}}$ extends to a homeomorphism $\left(v_{i}\right)^{-1}$ on $\operatorname{cl}\left(\right.$ int $\left.X_{i}\right)$ for $i \in I$ with int $X_{i} \neq \emptyset$. For notational convenience we denote $\left(v_{i}\right)^{-1}=\left.T\right|_{c l\left(\text { int } X_{i}\right)}$. Let $\underline{i}=\left(i_{1} \ldots i_{n}\right) \in I^{n}$ satisfy $\operatorname{int}\left(X_{i_{1}} \cap T^{-1} X_{i_{2}} \cap \ldots T^{-(n-1)} X_{i_{n}}\right) \neq \emptyset$. Then we define $X_{\underline{i}}:=X_{i_{1}} \cap T^{-1} X_{i_{2}} \cap \ldots \cap T^{-(n-1)} X_{i_{n}}$, which is called a cylinder of rank $n$ and write $|\underline{i}|=n$. By $(01),\left.T^{n}\right|_{\text {int } X_{i_{1} \ldots i_{n}}}: \operatorname{int} X_{i_{1} \ldots i_{n}} \rightarrow T^{n}\left(\operatorname{int}\left(X_{i_{1} \ldots i_{n}}\right)\right)$ is a homeomorphism and $\left(\left.T^{n}\right|_{\text {int } X_{i_{1} \ldots i_{n}}}\right)^{-1}$ extends to a homeomorphism $v_{i_{1}} \circ v_{i_{2}} \circ \ldots \circ v_{i_{n}}=$ $v_{i_{1} \ldots i_{n}}: \operatorname{cl}\left(T^{n}\left(\right.\right.$ int $\left.\left.X_{\underline{i}}\right)\right) \rightarrow \operatorname{cl}\left(\right.$ int $\left.X_{\underline{i}}\right)$ and $\left(v_{i_{1} \ldots i_{n}}\right)^{-1}=\left.T^{n}\right|_{c l\left(\text { int } X_{i_{1} \ldots i_{n}}\right)}$. We impose 
on $(T, X, Q)$ the next condition, which gives a nice countable states symbolic dynamics similar to sofic shifts (cf. [15], 16], [17, 19]):

(Finite Range Structure). $\mathcal{U}=\left\{\operatorname{int}\left(T^{n} X_{i_{1} \ldots i_{n}}\right): \forall X_{i_{1} \ldots i_{n}}, \forall n>0\right\}$ consists of finitely many open subsets $U_{1}, \ldots, U_{N}$ of $X$.

In particular, if $(T, X, Q)$ satisfies the Markov property (i.e., int $X_{i} \cap i n t T X_{j} \neq$ $\emptyset$ implies $\left.\operatorname{int} T X_{j} \supset \operatorname{int} X_{i}\right)$, then $\mathcal{U}=\left\{\operatorname{int}\left(T X_{i}\right): \forall i \in I\right\}$ and we say that $(T, X, Q)$ is an FRS Markov system. If $X_{i} \in Q$ satisfies $c l\left(T\left(\right.\right.$ int $\left.\left.X_{i}\right)\right)=X$, then $X_{i}$ is called a full cylinder. If all cylinders are full cylinders so that $\mathcal{U}=\{i n t X\}$, then $(T, X, Q)$ is called a Bernoulli system. We assume further the next transitive condition:

(Transitivity). int $X=\bigcup_{k=1}^{N} U_{k}$ and $\forall l \in\{1,2, \ldots, N\}, \exists 0<s_{l}<\infty$ such that for each $k \in\{1,2, \ldots, N\}, U_{k}$ contains an interior of a cylinder $X^{(k, l)}\left(s_{l}\right)$ of rank $s_{l}$ such that $T^{s_{l}}\left(\right.$ int $\left.X^{(k, l)}\left(s_{l}\right)\right)=U_{l}$.

The transitivity condition allows one to establish the next fact.

Lemma 1. There exists $0<S<\infty$ such that $T^{S}\left(\bigcup_{l=1}^{N}\right.$ int $\left.X^{(k, l)}\left(s_{l}\right)\right)=\operatorname{int} X$ and $\forall X_{i} \in Q, T^{S+1}\left(\right.$ int $\left.X_{i}\right)=$ int $X$.

Proof of Lemma 1. Since each $U_{k}$ contains $\bigcup_{l=1}^{N} \operatorname{int} X^{(k, l)}\left(s_{l}\right)$, choosing $S=\prod_{l=1}^{N} s_{l}$ is enough to establish the desired fact.

Remark (A). If $(T, X, Q)$ is a Markov system, then the transitivity condition implies aperiodicity in the following sense: $\exists S>0$ such that $\forall U_{k}, U_{l} \in \mathcal{U}, \forall n>S, \exists \underline{i}=$ $\left(i_{1} \ldots i_{n}\right)$ with $\operatorname{int} X_{\underline{i}} \neq \emptyset$ satisfying $\operatorname{int} X_{i_{1}} \subset U_{k}$ and $T\left(\operatorname{int}\left(X_{i_{n}}\right)\right)=U_{l}$.

Definition. We say that $\phi$ is a potential of weak bounded variation (WBV) if there exists a sequence of positive numbers $\left\{C_{n}\right\}$ satisfying $\lim _{n \rightarrow \infty}(1 / n) \log C_{n}=0$ and $\forall n \geq 1, \forall X_{i_{1} \ldots i_{n}} \in \bigvee_{j=0}^{n-1} T^{-j} Q$,

$$
\frac{\sup _{x \in X_{i_{1} \ldots i_{n}}} \exp \left(\sum_{j=0}^{n-1} \phi\left(T^{j} x\right)\right)}{\inf _{x \in X_{i_{1} \ldots i_{n}}} \exp \left(\sum_{j=0}^{n-1} \phi\left(T^{j} x\right)\right)} \leq C_{n} .
$$

Define

$$
\operatorname{Var}_{n}(T, \phi):=\sup _{Y \in \bigvee_{j=0}^{n-1} T^{-j}(Q)} \sup _{x, y \in Y}|\phi(x)-\phi(y)| .
$$

Remark (B). If $\operatorname{Var}_{n}(T, \phi) \rightarrow 0$ as $n \rightarrow \infty$, which implies continuity of $\phi$ in symbolic distance, then $\phi$ satisfies the WBV property. Hence if $(T, X, Q)$ is a subshift of finite type, then any continuous functions satisfy the WBV property and if $(T, X, Q)$ is a countable Markov shift, then any uniformly continuous functions $\phi$ with $\operatorname{Var}_{1}(T, \phi)<\infty$ satisfy the WBV property $([7)$.

Let $\mathcal{F}$ be the $\sigma$-algebra of Borel sets of the compact space $X$.

Definition ([17], [18], [20]). A probability measure $\nu$ on $(X, \mathcal{F})$ is called a weak Gibbs measure for a function $\phi$ with a constant $P$ if there exists a sequence $\left\{K_{n}\right\}_{n>0}$ of positive numbers with $\lim _{n \rightarrow \infty}(1 / n) \log K_{n}=0$ such that $\nu$-a.e. $x$,

$$
K_{n}^{-1} \leq \frac{\nu\left(X_{i_{1} \ldots i_{n}}(x)\right)}{\exp \left(\sum_{j=0}^{n-1} \phi T^{j}(x)+n P\right)} \leq K_{n},
$$

where $X_{i_{1} \ldots i_{n}}(x)$ denotes the cylinder containing $x$. 
For a function $\phi: X \rightarrow \mathbb{R}$, we define an operator $\mathcal{L}_{\phi}$ by

$$
\mathcal{L}_{\phi} g(x)=\sum_{i \in I} \exp \phi\left(v_{i}(x)\right) g\left(v_{i}(x)\right) 1_{c l\left(T\left(i n t X_{i}\right)\right)}(x)(\forall g \in C(X), \forall x \in X) .
$$

If $\phi$ satisfies $\operatorname{Var}_{n}(\phi) \rightarrow 0(n \rightarrow \infty),\left\|\mathcal{L}_{\phi} 1\right\|:=\sup _{x \in X} \mathcal{L}_{\phi} 1(x)<\infty$ and

(04) $\left\{v_{i}\right\}_{i \in I}$ is an equi-continuous family of partially defined uniformly continuous maps,

then $\mathcal{L}_{\phi}$ preserves $C(X)$ (i.e., $\left.\mathcal{L}_{\phi}: C(X) \rightarrow C(X)\right)$ and is called the RuellePerron-Frobenius operator. We remark that (04) is valid if $\sigma(n)=\sup \{\operatorname{diam} Y \mid Y \in$ $\left.\bigvee_{j=0}^{n-1} T^{-j}(Q)\right\} \rightarrow 0$ as $n \rightarrow \infty$.

We recall the next result, which follows from Theorem 5.1 in [17] and Proposition 2.2 in 18 .

Lemma 2 ([17, [18]). Let $(T, X, Q)$ be a transitive FRS Markov system satisfying int $X \in \mathcal{U}$, and let $\phi$ be a potential of $W B V$. Assume that there exist $p>0$ and a Borel probability measure $\nu$ on $(X, \mathcal{F})$ satisfying $\mathcal{L}_{\phi}^{*} \nu=p \nu$, where $\mathcal{L}_{\phi}^{*}$ is the dual of $\mathcal{L}_{\phi}$. Then $\nu$ is a weak Gibbs measure for $\phi$ with $-\log p$.

Definition. We say that a Borel probability measure $\nu$ on $X$ is an $f$-conformal measure if $\frac{\left.d(\nu T)\right|_{X_{i}}}{\left.d \nu\right|_{X_{i}}}=\left.f\right|_{X_{i}}(\forall i \in I)$ and $\nu\left(\bigcup_{i \in I} \partial X_{i}\right)=0$.

In order to show the weak Gibbs property of $\nu$, we use the following formula of the local Jacobians with respect to $\nu$ :

$$
\frac{\left.d(\nu T)\right|_{X_{i}}}{\left.d \nu\right|_{X_{i}}}=\left.\exp [\log p-\phi]\right|_{X_{i}}(\forall i \in I) .
$$

Thus for the existence of weak Gibbs measures, it is enough to show the existence of conformal measures (see $\S 3$ ).

Lemma 3 (Theorem 2.2 in [18]). Let $\nu$ be a weak Gibbs measure for $\phi$ with $-P$. If there exists a T-invariant ergodic probability measure $\mu$ equivalent to $\nu$ with $H_{\mu}(Q)<\infty$ and $\phi \in L^{1}(\mu)$, then $P=h_{\mu}(T)+\int_{X} \phi d \mu$.

In particular, if the constant $P$ is the measure-theoretical pressure, then the existence of a $T$-invariant ergodic probability measure $\mu$ equivalent to the weak Gibbs measure $\nu$ for $\phi$ with $-P$ implies the existence of an equilibrium state for $\phi$ (see $\S 4$ ). In order to achieve both constructions of conformal measures and equilibrium states, we need to introduce new derived systems which are called jump transformations ([13]). Let $B_{1} \subset X$ be a union of cylinders of rank 1 of which index $i$ belongs to a subset $J$ of $I$, and let $D_{1}:=B_{1}^{c}$. Define a function $R: X \rightarrow \mathbb{N} \cup\{\infty\}$ by $R(x)=\inf \left\{n \geq 0: T^{n} x \in B_{1}\right\}+1$ and for each $n>1$, define inductively

$$
B_{n}:=\{x \in X \mid R(x)=n\}, \quad D_{n}:=\{x \in X \mid R(x)>n\}\left(=\bigcap_{m=0}^{n-1} T^{-m} B_{1}^{c}\right) .
$$

Now we define Schweiger's jump transformation ([13]) $T^{*}: \bigcup_{n=1}^{\infty} B_{n} \rightarrow X$ by $T^{*} x=T^{R(x)} x$. We denote $X^{*}:=X \backslash\left(\bigcup_{m=0}^{\infty} T^{*-m}\left(\bigcap_{n \geq 0}\{R(x)>n\}\right)\right)$ and

$$
I^{*}:=\bigcup_{n \geq 1}\left\{\left(i_{1} \ldots i_{n}\right) \in I^{n}: X_{i_{1} \ldots i_{n}} \subseteq B_{n}\right\}
$$

Then it is easy to see that $\left(T^{*}, X^{*}, Q^{*}=\left\{X_{\underline{i}}\right\}_{\underline{\underline{i}} \in I^{*}}\right)$ is an FRS Markov system. For a given $\phi: X \rightarrow \mathbb{R}$, we define $\phi^{*}: \bigcup_{n=1}^{\infty} B_{n} \rightarrow \mathbb{R}$ by $\phi^{*}(x)=\sum_{h=0}^{R(x)-1} \phi T^{h}(x)$. 
Definition. We say that an FRS Markov system satisfies local exponential instability with respect to $B_{1}$ if (05): $\exists 0<\gamma^{*}<1, \exists 0<\Gamma^{*}<\infty$ such that $\forall n \geq 1$,

$$
\sigma_{X^{*}, T^{*}}(n)=\sup \left\{\operatorname{diam} Y \mid Y \in \bigvee_{j=0}^{n-1} T^{*-j}\left(Q^{*}\right)\right\} \leq \Gamma^{*} \gamma^{* n}
$$

Definition. We say that a potential $\phi: X \rightarrow \mathbb{R}$ satisfies local bounded distortion with respect to $B_{1}$ if there exists $\theta>0$ such that $(06): \forall \underline{i}=\left(i_{1} \ldots i_{|\underline{i}|}\right) \in I^{*}, \exists 0<$ $L_{\phi}(\underline{i})<\infty$ satisfying

$$
\left|\phi\left(v_{\underline{i}}(x)\right)-\phi\left(v_{\underline{i}}(y)\right)\right| \leq L_{\phi}(\underline{i}) d(x, y)^{\theta} \quad\left(\forall x, y \in T^{|\underline{\underline{i}}|} X_{\underline{i}}\right)
$$

and

$$
\sup _{\underline{i} \in I^{*}} \sum_{j=0}^{|\underline{i}|-1} L_{\phi}\left(i_{j+1} \ldots i_{|\underline{i}|}\right)<\infty .
$$

Under the conditions (05-06), we can easily verify that $\left\{\phi^{*} v_{\underline{i}}: \underline{i} \in I^{*}\right\}$ is an equi-Hölder continuous family (cf. [19], [20]) and $\sum_{n=1}^{\infty} \operatorname{Var}_{n}\left(T^{*}, \phi^{*}\right)<\infty$. Both conditions (05-06) can be easily verified for all higher-dimensional examples in $\S 8$.

In the rest of this section we shall state relations between jump transformations associated to $B_{1}$ and induced maps over $B_{1}$. Let $R_{B_{1}}: B_{1} \rightarrow \mathbb{R} \cup\{\infty\}$ be the first return function defined by $R_{B_{1}}(x)=\inf \left\{n \geq 1: T^{n} x \in B_{1}\right\}$. Then we define the induced map $T_{B_{1}}$ over $\left\{x \in B_{1}: R_{B_{1}}(x)<\infty\right\}$ by $T_{B_{1}} x=T^{R_{B_{1}}(x)} x$ and the induced potential $\phi_{B_{1}}:\left\{x \in B_{1}: R_{B_{1}}(x)<\infty\right\} \rightarrow \mathbb{R}$ by $\phi_{B_{1}}(x)=\sum_{h=0}^{R_{B_{1}}(x)-1} \phi T^{h}(x)$. Then we can immediately see the following facts.

Lemma 4. (4-1) $R_{B_{1}}=\left.R \circ T\right|_{B_{1}}$,

(4-2) $\phi_{B_{1}}-s R_{B_{1}}=\left.\left(\phi^{*}-s R\right) \circ T\right|_{B_{1}}+\left(\phi-\phi \circ T_{B_{1}}\right)$,

(4-3) $\sum_{m=0}^{n-1}\left(\phi_{B_{1}}-s R_{B_{1}}\right) \circ T_{B_{1}}^{m}=\left.\sum_{m=0}^{n-1}\left(\phi^{*}-s R\right) \circ T^{* m} \circ T\right|_{B_{1}{ }^{*}}+\left(\phi-\phi \circ T_{B_{1}}^{n}\right)$, where $B_{1}{ }^{*}:=B_{1} \backslash\left(\bigcup_{m=0}^{\infty} T_{B_{1}}^{-m}\left(\left\{R_{B_{1}}(x)=\infty\right\}\right)\right)$.

Lemma 5 (Lemma 4.1 in [18]). Suppose that $B_{1}$ consists of full cylinders. Then for any $T$-invariant probability measure $m$ with $m\left(B_{1}\right)>0, m_{B_{1}}:=\frac{\left.m\right|_{B_{1}}}{m\left(B_{1}\right)}$ is a $T_{B_{1}}$ invariant probability measure and $m^{*}:=\left.m_{B_{1}} T\right|_{B_{1}} ^{-1}$ is a $T^{*}$-invariant probability measure. $m$ can be written in terms of $m^{*}$ by Schweiger's formula (see (3) in §5) and in terms of $m_{B_{1}}$ by Kac's formula (see Lemma 16).

\section{§2. TOPOLOGICAL PRESSURE FOR POTENTIALS OF WEAK BOUNDED VARIATION}

Let $(T, X, Q)$ be a transitive FRS Markov system and let $\phi: X \rightarrow \mathbb{R}$ be a potential of WBV. For each $n>0$ and for each $U \in \mathcal{U}$ we define the following partition functions :

$$
\begin{gathered}
Z_{n}(U, \phi):=\sum_{\underline{i}:|\underline{i}|=n, \operatorname{int}\left(T X_{i_{n}}\right)=U \supset \operatorname{int} X_{i_{1}}} \sum_{v_{\underline{i}} x=x \in \operatorname{cl}\left(\operatorname{int} X_{\underline{i}}\right)} \exp \left[\sum_{h=0}^{n-1} \phi T^{h}(x)\right], \\
\bar{Z}_{n}(U, \phi)=\sum_{\underline{i}:|\underline{\mid}|=n, \operatorname{int}\left(T X_{i_{n}}\right)=U \supset \operatorname{int} X_{i_{1}}} \sup _{x \in X_{\underline{i}}} \exp \left[\sum_{h=0}^{n-1} \phi T^{h}(x)\right]
\end{gathered}
$$


and

We further define

$$
\underline{Z}_{n}(U, \phi)=\sum_{\underline{i}:|\underline{i}|=n, \operatorname{int}\left(T X_{i_{n}}\right)=U \supset \operatorname{int} X_{i_{1}}} \inf _{x \in X_{\underline{i}}} \exp \left[\sum_{h=0}^{n-1} \phi T^{h}(x)\right] .
$$

$$
Z_{n}(\phi):=\sum_{\underline{i}:|\underline{i}|=n, \operatorname{int}\left(T X_{i_{n}}\right) \supset \operatorname{int} X_{i_{1}}} \sum_{v_{\underline{i}} x=x \in c l\left(\operatorname{int} X_{\underline{i}}\right)} \exp \left[\sum_{h=0}^{n-1} \phi T^{h}(x)\right] .
$$

We shall define the topological pressure as the asymptotic growth rates of these partition functions.

Theorem 1 (Topological pressure for potentials of WBV). Let $(T, X, Q)$ be a transitive FRS Markov system and let $\phi$ be a potential of $W B V$. For each $U \in \mathcal{U}$, $\lim _{n \rightarrow \infty} \frac{1}{n} \log \bar{Z}_{n}(U, \phi), \lim _{n \rightarrow \infty} \frac{1}{n} \log \underline{Z}_{n}(U, \phi), \lim _{n \rightarrow \infty} \frac{1}{n} \log Z_{n}(U, \phi)$ exist and do not depend on $U$. Furthermore, the limits coincide with $\lim _{n \rightarrow \infty} \frac{1}{n} \log Z_{n}(\phi)$.

We call the limit $P_{\text {top }}(T, \phi):=\lim _{n \rightarrow \infty} \frac{1}{n} \log Z_{n}(\phi)$ the topological pressure for $\phi$. The next fact can be verified easily.

Lemma 6. Under the next condition, $Z_{n}(\phi)$ coincides with the usual partition function defined by : $\sum_{T^{n} x=x, x \in X} \exp \left[\sum_{h=0}^{n-1} \phi T^{h}(x)\right]$.

$$
\begin{aligned}
& \text { For } x_{0} \in X_{i_{1} \ldots i_{n}} \text { with } T^{n} x_{0}=x_{0} \text {, either } x_{0} \in \operatorname{int} X_{i_{1} \ldots i_{n}} \text { or } x_{0} \notin c l X_{j_{1} \ldots j_{n}} \\
& \text { for }\left(j_{1} \ldots j_{n}\right) \neq\left(i_{1} \ldots i_{n}\right) \text {. }
\end{aligned}
$$

Let $\mathcal{V}$ be the finite disjoint partition generated by $\mathcal{U}$. We should claim that if a periodic point $x_{0}$ with period $n$ is contained in a cylinder $X_{i_{1} \ldots i_{n}}$ satisfying $X_{i_{1} \ldots i_{n}} \subset$ int $V$ for some $V \in \mathcal{V}$, then $x_{0} \notin \partial X_{i_{1} \ldots i_{n}}$. If not, we have a contradiction to $x_{0} \in$ int $V$ because of $x_{0} \in T^{n}\left(\partial X_{i_{1} \ldots i_{n}}\right)=\partial\left(T^{n} X_{i_{1} \ldots i_{n}}\right)$. By using this fact, we will see that all higher-dimensional examples in $\S 8$ satisfy (1). The Artin-MazurRuelle zeta function $\zeta_{T, \phi}(z)$ is defined by $\zeta_{T, \phi}(z)=\exp \left[\sum_{n=1}^{\infty} \frac{z^{n}}{n} Z_{n}(\phi)\right]$. Then the radius of convergence of $\zeta_{T, \phi}(z)$ is given by $\rho_{\phi}=\exp \left[\lim \sup _{n \rightarrow \infty} \frac{1}{n} \log Z_{n}(\phi)\right]^{-1}$. We define

$$
\mathcal{W}(T):=\left\{\phi: X \rightarrow \mathbb{R} \mid \phi \text { satisfies WBV and } P_{\text {top }}(T, \phi)<\infty\right\}
$$

and

$$
\mathcal{W}_{B}(T):=\left\{\phi \in \mathcal{W}(T)\left|\operatorname{Var}_{n} \phi \rightarrow 0(n \rightarrow 0),\|\phi\|:=\sup _{x \in X}\right| \phi(x) \mid<\infty\right\} .
$$

We can easily see that the pressure function $P_{\text {top }}(T,):. \mathcal{W}(T) \rightarrow \mathbb{R}$ satisfies continuity, convexity and $\forall \phi_{1}, \phi_{2} \in \mathcal{W}(T), P_{\text {top }}\left(T, \phi_{1}+\phi_{2}\right) \leq P_{\text {top }}\left(T, \phi_{1}\right)+P_{\text {top }}\left(T, \phi_{2}\right)$. Furthermore, by applying Theorem 2.4 in [7] we have the following fact.

Theorem 2. $\mathcal{W}_{B}(T)$ is a Banach space and $P_{\mathrm{top}}(T,):. \mathcal{W}_{B}(T) \rightarrow \mathbb{R}$ is a Lipschitz continuous convex function.

Definition. If an FRS Markov system $(T, X, Q)$ satisfies that $\forall U \in \mathcal{U}, \exists X_{i} \in Q$ such that $X_{i} \subset U$ and $T\left(i n t X_{i}\right)=i n t X$, then $(T, X, Q)$ is called a strongly transitive FRS Markov system.

Theorem 3 (Topological pressure and the spectral radius). Let $(T, X, Q)$ be a strongly transitive FRS Markov system satisfying $\mathcal{U} \cap \mathcal{V} \neq \emptyset$. Let $\phi$ be a potential of weak bounded variation. Then $\forall U \in \mathcal{U} \cap \mathcal{V}$ and $\forall x \in U, \lim _{n \rightarrow \infty} \frac{1}{n} \log \mathcal{L}_{\phi}^{n} 1_{U}(x)=$ $P_{\text {top }}(T, \phi)$. Futhermore, $\lim _{n \rightarrow \infty} \frac{1}{n} \log \left\|\mathcal{L}_{\phi}^{n} 1\right\|=\lim _{n \rightarrow \infty} \frac{1}{n} \log \left\|\mathcal{L}_{\phi}^{n}\right\|=P_{\text {top }}(T, \phi)$. 
We can easily verify all conditions in Theorem 3 for examples in $\S 8$.

\section{§3. The construction of weak Gibbs conformal measures}

Let $(T, X, Q)$ be a transitive FRS Markov system and let $\phi \in \mathcal{W}(T)$. Suppose that there exists a union of full cylinders $B_{1}(\subset X)$ with respect to which $(T, X, Q)$ satisfies local exponential instability and $\phi$ satisfies local bounded distortion. For the derived potential $\phi^{*}(x)=\sum_{h=0}^{R(x)-1} \phi T^{h}(x)$ we define

$$
Z_{n}\left(\phi^{*}\right):=\sum_{\left(\underline{i}_{1} \ldots \underline{i}_{n}\right) \in I^{* n}: \operatorname{int}\left(T^{*} X_{\underline{i}_{n}}\right) \supset \operatorname{int} X_{\underline{i}_{1}}} \sum_{\underline{v}_{1} \ldots \underline{i}_{n}} \sum_{x=x \in c l\left(\operatorname{int} X_{\underline{i}_{1} \ldots \underline{i}_{n}}\right)} \exp \left[\sum_{m=0}^{n-1} \phi T^{* m}(x)\right] .
$$

Then by Theorem 1 , summable variations of $\phi^{*}$ allow one to show that

$$
\exists \lim _{n \rightarrow \infty} \frac{1}{n} \log Z_{n}\left(\phi^{*}\right):=P_{\text {top }}\left(T^{*}, \phi^{*}\right) \in(-\infty, \infty] .
$$

Theorem 4 (A construction of conformal measures via jump transformations). Let $(T, X, Q)$ be a transitive FRS Markov system and let $\phi \in \mathcal{W}(T)$. Suppose that there exists a union of full cylinders $B_{1}(\subset X)$ with respect to which $(T, X, Q)$ satisfies local exponential instability and $\phi$ satisfies local bounded distortion. Assume further that $\left\|\mathcal{L}_{\phi^{*}-R \min \left\{0, P_{\mathrm{top}}\left(T^{*}, \phi^{*}\right)\right\}} 1\right\|<\infty$. Then there exists a Borel probability measure $\nu$ on $X$ supported on $X^{*}$ satisfying

$$
\left.\frac{d \nu T}{d \nu}\right|_{X_{i}}=\exp \left[P_{\text {top }}(T, \phi)-\phi\right](\forall i \in I)
$$

and $\nu\left(\bigcup_{i \in I} \partial X_{i}\right)=0$.

As we have announced in $\S 0$, for constructing a weak Gibbs measure for $\phi$ of WBV, we shall consider the following generalized Bowen's equation:

$$
(\mathrm{GBE}): P_{\mathrm{top}}\left(T^{*}, \phi^{*}-s R\right)=0 .
$$

The existence of a zero of the equation (GBE) follows from the standard argument in the case when $0 \leq P_{\text {top }}\left(T^{*}, \phi^{*}\right)<\infty$ because of continuity of the function $s \rightarrow P_{\text {top }}\left(T^{*}, \phi^{*}-s R\right)$ on int $\left\{s \in \mathbb{R} \mid P_{\text {top }}\left(T^{*}, \phi^{*}-s R\right) \in \mathbb{R}\right\}$ (see Lemma 14). We should notice that the uniqueness of the zero of (GBE) follows from the "strictly" decreasing property of the function, $s \rightarrow P_{\mathrm{top}}\left(T^{*}, \phi^{*}-s R\right)$ in the standard situation. Here we have no evidence of it although the function is decreasing. If $P_{\text {top }}\left(T^{*}, \phi^{*}\right)<$ 0 , then under the assumption $P_{\text {top }}\left(T^{*}, \phi^{*}-R P_{\text {top }}\left(T^{*}, \phi^{*}\right)\right)<\infty$ we see that

$$
P_{\text {top }}\left(T^{*}, \phi^{*}-R P_{\text {top }}\left(T^{*}, \phi^{*}\right)\right) \geq P_{\text {top }}\left(T^{*}, \phi^{*}-P_{\text {top }}\left(T^{*}, \phi^{*}\right)\right)=0
$$

and so we can reduce to the previous case. If $P_{\text {top }}\left(T^{*}, \phi^{*}\right)=\infty$, then we cannot use the standard argument. Now we come to state the next key lemma, which allows one to establish Theorem 4.

Lemma 7 (The existence of a zero of (GBE)). (i) If $0 \leq P_{\text {top }}\left(T^{*}, \phi^{*}\right)<\infty$, then $P_{\text {top }}(T, \phi) \geq 0$ and $\exists s_{0} \geq 0$ satisfying $P_{\text {top }}\left(T^{*}, \phi^{*}-s_{0} R\right)=0$.

(ii) If $P_{\text {top }}\left(T^{*}, \phi^{*}\right)<0$ and $P_{\text {top }}\left(T^{*}, \phi^{*}-R P_{\text {top }}\left(T^{*}, \phi^{*}\right)\right)<\infty$, then $P_{\text {top }}(T, \phi-$ $\left.P_{\text {top }}\left(T^{*}, \phi^{*}\right)\right) \geq 0$ and $\exists s_{0} \geq 0$ satisfying $P_{\text {top }}\left(T^{*},\left(\phi-P_{\text {top }}\left(T^{*}, \phi^{*}\right)\right)^{*}-s_{0} R\right)=0$.

(iii) If $\sup \left\{s \in \mathbb{R}: P_{\text {top }}\left(T^{*}, \phi^{*}-s R\right)=\infty\right\}=\min \left\{0, P_{\text {top }}\left(T^{*}, \phi^{*}\right)\right\}$, then $\exists s_{0} \geq$ $\min \left\{0, P_{\text {top }}\left(T^{*}, \phi^{*}\right)\right\}$ such that $P_{\text {top }}\left(T^{*}, \phi^{*}-s_{0} R\right)=0$. 
We recall the formal power series $\zeta_{T, \phi}(z)=\exp \left[\sum_{n=1}^{\infty} \frac{z^{n}}{n} Z_{n}(\phi)\right]$, which is called the Artin-Mazur-Ruelle zeta function. The next product formula of zeta functions plays an important role in proving Lemma 7.

Proposition 1 (cf. [17]). We can write

$$
\zeta_{T, \phi}(\exp (-s))=\zeta_{T^{*}, \phi^{*}-s R}(1) \times \zeta_{\left.T\right|_{\cap_{n}>0} D_{n}, \phi}(\exp (-s)) .
$$

Corollary 1. If $s>P_{\text {top }}(T, \phi)$, then $P_{\text {top }}\left(T^{*}, \phi^{*}-s R\right) \leq 0$.

By Theorem 3, the assumption $\left\|\mathcal{L}_{\phi^{*}-R \min \left\{0, P_{\text {top }}\left(T^{*}, \phi^{*}\right)\right\}} 1\right\|<\infty$ implies either $0 \leq P_{\text {top }}\left(T^{*}, \phi^{*}\right)<\infty$ or $P_{\text {top }}\left(T^{*}, \phi^{*}-R P_{\text {top }}\left(T^{*}, \phi^{*}\right)\right)<\infty$ is satisfied. Hence it follows from Lemma 7 that $\exists s_{0} \geq \min \left\{0, P_{\text {top }}\left(T^{*}, \phi^{*}\right)\right\}$ satisfying $P_{\text {top }}\left(T^{*}, \phi^{*}-\right.$ $\left.s_{0} R\right)=0$ and $\left\|\mathcal{L}_{\phi^{*}-s_{0} R} 1\right\|<\infty$. Since $Q^{*}=\left\{X_{\underline{i}}\right\}_{\underline{i} \in I^{*}}$ consists of full cylinders and summability of variations $\sum_{n=1}^{\infty} \operatorname{Var}_{n}\left(T^{*}, \phi^{*}-s_{0} R\right)<\infty$ is valid, we can apply P. Walter's argument in [14] to show the existence of an $\exp \left[s_{0} R-\phi^{*}\right]$-conformal measure with respect to $T^{*}$.

Lemma 8. There exists a Borel probability measure $\nu$ on $X$ satisfying $\mathcal{L}_{\phi^{*}-s_{0}}^{*} \nu=$ $\nu$ and $\nu\left(\right.$ int $\left.X^{*}\right)=1$.

In $\S 9$ we shall show the existence of an $\exp \left[s_{0}-\phi\right]$-conformal measure for the zero $s_{0}$ of (GBE) by using the conformal measure $\nu$ on $X^{*}$ and show $s_{0}=P_{\text {top }}(T, \phi)$, which implies uniqueness of the zero of $(\mathrm{GBE})$. At the end of this section, we shall consider the case when $\left\|\mathcal{L}_{\phi^{*}-R \min \left\{0, P_{\text {top }}\left(T^{*}, \phi^{*}\right)\right\}} 1\right\|=\infty$. By Theorem 3, if $P_{\text {top }}\left(T^{*}, \phi^{*}-s_{0} R\right)=0$, then there exists sufficiently large $n$ such that $\left\|\mathcal{L}_{\left(\phi-s_{0}\right)^{*}}^{n} 1\right\|=$ $\left\|\mathcal{L}_{\left(\phi-s_{0}\right)_{n}^{*}} 1\right\|<\infty$, where

$$
\left(\phi-s_{0}\right)_{n}^{*}:=\sum_{m=0}^{n-1}\left(\phi-s_{0}\right)^{*} T^{* m}=\sum_{m=0}^{n-1}\left(\phi^{*}-s_{0} R\right) T^{* m} .
$$

We shall introduce a new stopping time (depending on $n \geq 1$ ) defined on $X^{*}$ by

$$
R_{n}(x):=\inf \left\{k \geq n \mid X_{i_{1} \ldots i_{k}}(x) \in \bigvee_{m=0}^{n-1} T^{*-m} Q^{*}\right\}=\sum_{m=0}^{n-1} R\left(T^{* m}(x)\right)
$$

Then a new jump transformation $S^{*}$ defined by $S^{*}(x):=T^{R_{n}(x)}(x)$ is equal to $T^{* n}$ and the next facts can be verified easily.

Lemma 9. (9-1) $R_{n}(T(x))=R_{n}(x)-1$.

(9-2) $\left\{x \in X^{*} \mid R_{n}(x)=k\right\}=\left\{x \in X^{*}\left|\sum_{m=1}^{n}\right| \underline{i_{m}} \mid=k\right.$ where $x \in X_{\underline{i_{1}} \ldots \underline{i_{n}}} \in$ $\left.\bigvee_{m=0}^{n-1} T^{*-m} Q^{*}\right\}$

$(9-3)\left(\phi-s_{0}\right)_{n}^{*}(x)=\phi_{n}^{*}(x)-s_{0} R_{n}(x)=\sum_{h=0}^{R_{n}(x)-1}\left(\phi-s_{0}\right) T^{h}(x)$.

Now we shall consider a two-parameter family of functions $\left\{(\phi-s)_{n}^{*} \mid(s, n) \in\right.$ $\mathbb{R} \times \mathbb{N}\}$ and the equations $P_{\text {top }}\left(T^{* n},(\phi-s)_{n}^{*}\right)=0$. Applying Theorem 4 gives the next result.

Proposition 2. Suppose that all conditions in Theorem 3 are satisfied. If there exist $s_{0} \in \mathbb{R}$ and $n_{0} \in \mathbb{N}$ such that $\forall n \geq n_{0}, P_{\text {top }}\left(T^{* n},\left(\phi-s_{0}\right)_{n}^{*}\right)=0$, then there exists a Borel probability measure $\nu$ on $X$ supported on $X^{*}$ satisfying $\left.\frac{d \nu T}{d \nu}\right|_{X_{i}}=$ $\exp \left[s_{0}-\phi\right](\forall i \in I)$ and $\nu\left(\bigcup_{i \in I} \partial X_{i}\right)=0$. Furthermore, $s_{0}=P_{\text {top }}(T, \phi)$. 


\section{§4. INDIFFERENT PERIODIC POINTS AND NON-GIBBSIANNESS}

Let $(T, X, Q)$ be a transitive FRS Markov system and let $\phi \in \mathcal{W}(T)$. The next lemma follows from the definition of $P_{\text {top }}(T, \phi)$ directly.

Lemma 10. $P_{\text {top }}(T, \phi) \geq \frac{1}{q} \sum_{h=0}^{q-1} \phi T^{h}\left(x_{0}\right)\left(\forall x_{0} \in X, T^{q} x_{0}=x_{0}\right)$.

Definition. $x_{0}$ is called a generalized indifferent periodic point with period $q$ with respect to $\phi$ if $P_{\text {top }}(T, \phi)=\frac{1}{q} \sum_{h=0}^{q-1} \phi T^{h}\left(x_{0}\right)$. If $x_{0}$ is not indifferent, then we call $x_{0}$ a generalized repelling periodic point.

If a potential $\phi$ of WBV admits a generalized indifferent periodic point, then we can observe interesting statistical phenomena. More specifically, if there exists an $\exp \left[P_{\text {top }}(T, \phi)-\phi\right]$-conformal measure $\nu$, then the above definitions can be described in terms of the local Jacobians with respect to $\nu$, that is,

$$
\left.\frac{d\left(\nu T^{q}\right)}{d \nu}\right|_{X_{i_{1} \ldots i_{q}}\left(x_{0}\right)}\left(x_{0}\right)=\exp \left[q P_{\text {top }}(T, \phi)-\sum_{h=0}^{q-1} \phi T^{h}\left(x_{0}\right)\right]=1 .
$$

Then we have the following facts.

Proposition 3. Let $x_{0}$ be a generalized indifferent periodic point with period $q$ with respect to $\phi \in \mathcal{W}(T)$. Let $\nu$ be an $\exp \left[P_{\text {top }}(T, \phi)-\phi\right]$-conformal measure. Then

(i) $\forall s \geq 1, P_{\text {top }}(T, s \phi)=s P_{\text {top }}(T, \phi)$ and $\forall s<1, P_{\text {top }}(T, s \phi) \geq s P_{\text {top }}(T, \phi)$.

(ii) $\nu\left(X_{i_{1} \ldots i_{n}}\left(x_{0}\right)\right)$ decays subexponentially fast.

Proof. By Lemma 10, we have $P_{\text {top }}(T, s \phi) \geq s \frac{1}{q} \sum_{i=0}^{q-1} \phi T^{i}\left(x_{0}\right)$. In particular, if $x_{0}$ is a generalized indifferent periodic point for $\phi$, then $P_{\text {top }}(T, s \phi) \geq s P_{\text {top }}(T, \phi)$. We recall that by Lemma 2 the conformal measure $\nu$ is a weak Gibbs measure for $\phi$ of WBV. Then we have for $s \geq 1$,

$$
\frac{1}{n} \log Z_{n}(s \phi) \leq s P_{\text {top }}(T, \phi)+\frac{1}{n} \log C_{n} K_{n}^{s}+\frac{1}{n} \log \sum_{i_{1} \ldots i_{n}} \nu\left(X_{i_{1} \ldots i_{n}}\right)^{s},
$$

where both $C_{n}$ and $K_{n}$ satisfy $\lim _{n \rightarrow \infty} \frac{1}{n} \log C_{n}=0$ and $\lim _{n \rightarrow \infty} \frac{1}{n} \log K_{n}=0$. Since $\lim _{n \rightarrow \infty} \frac{1}{n} \log C_{n} K_{n}^{s}=0$, we have $P_{\text {top }}(T, s \phi) \leq s P_{\text {top }}(T, \phi)$ for $s \geq 1$. (ii) follows from Proposition 6.1 in [21].

Let us recall that $\nu$ was obtained by constructing a jump transformation in Theorem 4. Then we can associate the generalized indifferent periodic points to the marginal sets $\bigcap_{n \geq 0} D_{n}$.

Proposition 4. Let $x_{0}$ be a generalized indifferent periodic point with period $q$ with respect to $\phi \in \mathcal{W}(T)$.

(i) (Failure of bounded distortion)

$$
C_{n q}\left(x_{0}\right):=\sup _{x, y \in X_{i_{1} \ldots i_{n q}\left(x_{0}\right)}} \frac{\exp \left[\sum_{h=0}^{n q-1} \phi T^{h}(x)\right]}{\exp \left[\sum_{h=0}^{n q-1} \phi T^{h}(y)\right]} \rightarrow \infty
$$

monotonically as $n \rightarrow \infty$.

(ii) $x_{0} \in \bigcap_{n \geq 0} D_{n}$. 
Proof. Since $C_{n}\left(x_{0}\right)$ is the distortion of $\frac{d\left(\nu T^{n}\right)}{d \nu}$ over cylinders $X_{i_{1} \ldots i_{n}}\left(x_{0}\right)$, (i) follows from Lemma 6.1 in [21]. Suppose $x_{0} \notin \bigcap_{n>0} D_{n}$. Then by Sublemma A (see $\S 9$ ) we have $x_{0} \in X^{*}$. Since $\sum_{n=1}^{\infty} \operatorname{Var}_{n}\left(T^{*}, \phi^{*}\right)<\infty$ implies that $C_{n q}\left(x_{0}\right)$ cannot increase monotonically, we have a contradiction to (i). We complete the proof.

Remark $(C)$. We claim that $\bigcap_{n \geq 0} D_{n}$ can contain repelling periodic points.

If we have a $T$-invariant probability measure $\mu$ equivalent to $\nu$ via Kac's formula (Lemma 16) or Schweiger's formula (3) in $\S 5$, then the invariant densities $d \mu / d \nu$ are typically unbounded at indifferent periodic points with respect to $\nu$ (Lemma 6.2 in [21]) so that we can see interesting phenomena from a statistical point of view ([19], 21]). For example, under the existence of a generalized indifferent periodic point $x_{0}$ with respect to $\phi$, the rate of decay of correlation may be slower than $\nu\left(X_{i_{1} \ldots i_{n}}\left(x_{0}\right)\right)$, which decays subexonentially fast by (ii) in Proposition 3 . We referee 21] for further details. On the other hand, the Dirac measure $m$ supported on the generalized indifferent periodic orbit with respect to $\phi$ satisfies $P_{\text {top }}(T, \phi)=$ $h_{m}(T)+\int_{X} \phi d m$. Hence if we can establish a variational principle for the topological pressure and can construct a $T$-invariant measure $\mu$ equivalent to the weak Gibbs measure $\nu$ for $\phi$ with $-P_{\text {top }}(T, \phi)$, then by Lemma 3 we see immediately failure of uniqueness of equilibrium states. Furthermore, by the definition of indifferency we can show failure of Gibbsianness of equlibrium states for $\phi$ with generalized indifferent periodic points.

Theorem 5 (Characterization of non-Gibbsianness). Suppose that a potential $\phi$ with $P_{\mathrm{top}}(T, \phi)<\infty$ admits a generalized indifferent periodic point $x_{0}$. Then there is no Borel probability measure that is Gibbs for $\phi$.

\section{§5. Equilibrium STATES FOR POTENTIALS OF WEAK BOUNDED VARIATION}

Let $(T, X, Q)$ be a transitive FRS Markov system and let $M_{T}(X)$ be the set of all $T$-invariant probability measures on $(X, \mathcal{F})$. For $m \in M_{T}(X), I_{m}$ denotes the conditional information of $Q$ with respect to $T^{-1} \mathcal{F}$. We denote

$$
\begin{gathered}
M_{T}(X, \phi):=\left\{m \in M_{T}(X) \mid I_{m}+\phi \in L^{1}(m), \text { either } h_{m}(T)<\infty\right. \text { or } \\
\left.\int_{X} \phi d m>-\infty \text { is satisfied }\right\} .
\end{gathered}
$$

Theorem 6. Let $(T, X, Q)$ be a transitive FRS Markov system and let $\phi \in \mathcal{W}(T)$. Suppose that there exists a union of full cylinders $B_{1}(\subset X)$ with respect to which $(T, X, Q)$ satisfies local exponential instability and $\phi$ satisfies local bounded distortion. Let $\nu$ be the $\exp \left[P_{\text {top }}(T, \phi)-\phi\right]$-conformal measure supported on $X^{*}$. Assume further that $\Gamma:=\bigcap_{n \geq 0} D_{n}$ consists of periodic points. If $\int_{X^{*}} R d \nu<\infty$ and $H_{\nu}\left(Q^{*}\right)<\infty$, then there exists a T-invariant ergodic probability measure $\mu$ equivalent to $\nu$ that satisfies the following variational principle:

$P_{\text {top }}(T, \phi)=h_{\mu}(T)+\int_{X} \phi d \mu=\sup \left\{h_{m}(T)+\int_{X} \phi d m \mid m \in M_{T}(X, \phi)\right.$ is ergodic $\}$.

If $E_{T}(X, \phi):=\left\{m \in M_{T}(X, \phi) \mid h_{m}(T)+\int_{X} \phi d m=P_{\text {top }}(T, \phi)\right\}$ contains at least two elements, then it implies physically coexistence of different phases, which is so-called "phase transition". Phase transition may be related to failure of the Gibbs property of equilibrium states (see Theorem 5). 
Corollary 2 (Phase transition). We assume all conditions in Theorem 6 . If $\Gamma$ consists of generalized indifferent periodic points with respect to $\phi$, then the set of equilibrium states for $\phi$ is the convex hull of $\mu$ and the set of invariant Borel probability measures supported on $\Gamma$.

In order to prove Theorem 6 , we need a sequence of lemmas. Let $M_{T^{*}}\left(X^{*}\right)$ denote the set of all Borel probability measures on $X^{*}$ invariant under $T^{*}$. For $\phi^{*}-s R$ we define

$$
\begin{gathered}
M_{T^{*}}\left(X^{*}, \phi^{*}-s R\right):=\left\{m^{*} \in M_{T^{*}}\left(X^{*}\right) \mid \text { either } h_{m^{*}}\left(T^{*}\right)<\infty\right. \text { or } \\
\left.\int_{X^{*}}\left(\phi^{*}-s R\right) d m^{*}>-\infty \text { is satisfied }\right\} .
\end{gathered}
$$

Let $s_{0}=P_{\text {top }}(T, \phi)$. Then P. Walter's method in [14 can apply for $T^{*}$ and for $\phi^{*}-s_{0} R$ so that there exists the unique equlibrium state $\mu^{*}$ equivalent to $\nu$ and the following variational principle is valid:

$$
\begin{aligned}
0 & =P_{\text {top }}\left(T^{*}, \phi^{*}-s_{0} R\right)=h_{\mu^{*}}\left(T^{*}\right)+\int_{X^{*}}\left(\phi^{*}-s_{0} R\right) d \mu^{*} \\
& =\sup \left\{m^{*} \in M_{T^{*}}\left(X^{*}, \phi^{*}-s_{0} R\right) \mid h_{m^{*}}\left(T^{*}\right)+\int_{X^{*}}\left(\phi^{*}-s_{0} R\right) d m^{*}\right\} .
\end{aligned}
$$

Since $\sum_{n=1}^{\infty} \operatorname{Var}_{n}\left(T^{*}, \phi^{*}-s_{0} R\right)<\infty$ implies the bounded distortion property with respect to $\nu$ :

$$
\sup _{n \geq 1} \sup _{Y \in \bigvee_{j=0}^{n-1} T^{*-j}\left(Q^{*}\right)} \sup _{x, x^{\prime} \in Y} \frac{\left.\frac{d\left(\nu T^{* n}\right)}{d \nu}\right|_{Y}(x)}{\left.\frac{d\left(\nu T^{* n}\right)}{d \nu}\right|_{Y}\left(x^{\prime}\right)}<\infty,
$$

we can show ergodicity and Bowen's Gibbs property for $\mu^{*}$. If $\int_{X^{*}} R d \mu^{*}<\infty$, then the next Schweiger's formula ([13) gives a $T$-invariant ergodic probability measure $\mu$ equivalent to $\nu$ that satisfies $\mu\left(B_{1}\right)=\left(\int_{X^{*}} R d \mu^{*}\right)^{-1}>0$ :

$$
\left(\int_{X^{*}} R d \mu^{*}\right) \mu(E)=\sum_{n=0}^{\infty} \mu^{*}\left(D_{n} \cap T^{-n} E\right)=\int_{X^{*}} \sum_{i=0}^{R(x)-1} 1_{E} T^{i}(x) d \mu^{*}(x)
$$

and by Lemma 5 for $f \in L^{1}(\mu)$,

$$
\int_{X} f d \mu=\frac{\int_{X^{*}} \sum_{i=0}^{R(x)-1} f T^{i}(x) d \mu^{*}}{\int_{X^{*}} R d \mu^{*}}=\frac{\int_{B_{1}} \sum_{i=0}^{R_{B_{1}}(x)-1} f T^{i}(x) d \mu_{B_{1}}}{\int_{B_{1}} R_{B_{1}} d \mu_{B_{1}}}
$$

(cf. Lemma 4.2 in [18]). Since $\int_{X^{*}} R d \mu^{*}<\infty$ gives the equality

$$
\int_{X^{*}}\left(\phi^{*}-s_{0} R\right) d \mu^{*}=\int_{X^{*}} \phi^{*} d \mu^{*}-s_{0} \int_{X^{*}} R d \mu^{*}
$$

and $H_{\mu^{*}}\left(Q^{*}\right)<\infty$ gives $h_{\mu}(T)<\infty$, we can establish the following characterization of the zero $s_{0}$ of (GBE).

Lemma 11. If $\mu^{*} \in M_{T^{*}}\left(X^{*}\right)$ is ergodic and satisfies $h_{\mu^{*}}\left(T^{*}\right)+\int_{X^{*}}\left(\phi^{*}-s_{0} R\right) d \mu^{*}$ $=0, \int_{X^{*}} R d \mu^{*}<\infty$ and $H_{\mu^{*}}\left(Q^{*}\right)<\infty$, then

$$
s_{0}=\frac{h_{\mu^{*}}\left(T^{*}\right)+\int_{X^{*}} \phi^{*} d \mu^{*}}{\int_{X^{*}} R d \mu^{*}}=h_{\mu}(T)+\int_{X} \phi d \mu,
$$

where $\mu$ is obtained by formula (3). 
By Lemma 11 we have a $T$-invariant ergodic probability measure $\mu$ equivalent to $\nu$ that satisfies $P_{\text {top }}(T, \phi)=h_{\mu}(T)+\int_{X} \phi d \mu$.

Lemma 12 (Lemma 4.4 in [18]). If a T-invariant probability measure $m$ satisfies $m\left(B_{1}\right)=0$, then $\Gamma:=\bigcap_{n \geq 0} D_{n}$ is a full measure set with respect to $m$.

Proof of Theorem 6. By Lemma 11 for all $T$-invariant ergodic probability measures $m$ on $X$ with $m\left(B_{1}\right)>0$ and $m \in M_{T}(X, \phi)$, we can establish

$$
0=\frac{h_{\mu}(T)+\int_{X} \phi d \mu-P_{\mathrm{top}}(T, \phi)}{\mu\left(B_{1}\right)} \geq \frac{h_{m}(T)+\int_{X} \phi d m-P_{\mathrm{top}}(T, \phi)}{m\left(B_{1}\right)} .
$$

On the other hand, by Lemma 12 , any $T$-invariant ergodic probability measure $m$ on $X$ with $m\left(B_{1}\right)=0$ satisfies $m(\Gamma)=1$. In particular, if $\Gamma:=\bigcap_{n \geq 0} D_{n}$ consists of periodic points, then $h_{m}(T)+\int_{X} \phi d m=\int_{\Gamma} \phi d m \leq P_{\text {top }}(T, \phi)$, which completes the proof of Theorem 6 .

\section{$\S 6$. The construction of $\sigma$-Finite Conformal measures VIA INDUCED MAPS}

Let $(T, X, Q)$ be a transitive FRS Markov system and $\phi \in \mathcal{W}(T)$. Suppose that $(T, X, Q)$ satisfies local exponential instability and $\phi$ satisfies local bounded distortion with respect to $B_{1}=\bigcup_{j \in J} X_{j}(J \subset I)$. Let $A_{j}=\operatorname{cl}\left(\right.$ int $\left.X_{j}\right)$ for $j \in J$ and put $A:=\bigcup_{j \in J} A_{j}$. We define the first return function $R_{A}: A \rightarrow \mathbb{R} \cup\{\infty\}$ and the induced map $T_{A}$ over $\left\{x \in A: R_{A}(x)<\infty\right\}$. By the Markov property, there exists a partition of the set $B_{k}^{(A)}=\left\{x \in A: R_{A}(x)=k\right\}$ for each $k \geq 1$ so that $T^{k}$ restricted to the interior of each element of the partition is a homeomorphism onto its image. $I_{A}$ denotes the set of all indices corresponding to such elements of the partition of $\bigcup_{k \geq 1} B_{k}^{(A)}$. Then $\left\{v_{\underline{i}}: \underline{i} \in I_{A}\right\}$ is a family of extensions of local inverses of $T_{A}$. For $s \in \mathbb{R}$ and $x \in \bigcup_{k=1}^{\infty} B_{k}^{(A)}$ we define $\phi_{A}^{(s)}(x)=\sum_{h=0}^{R_{A}(x)-1} \phi T^{h}(x)-s R_{A}(x)$. By Lemma 4 we can easily see the next fact.

Lemma 13. If each $A_{i} \subset A$ satisfies $T A_{i}=X$, then

$$
P_{\text {top }}\left(T^{*}, \phi^{*}-s R\right)=P_{\text {top }}\left(T_{A}, \phi_{A}^{(s)}\right) .
$$

We recall the following result in [6].

Lemma $14([6])$. If $\left\|\mathcal{L}_{\phi_{A}^{(0)}} 1\right\|<\infty$, then $s \rightarrow P_{\text {top }}\left(T_{A}, \phi_{A}^{(s)}\right)$ is continuous on $\operatorname{int}\left\{s \in \mathbb{R}: P_{\text {top }}\left(T_{A}, \phi_{A}^{(s)}\right) \in \mathbb{R}\right\}$.

We suppose that $B_{1}$ consists of a single full cylinder $X_{j}$ and the following conditions are satisfied for $A=\operatorname{cl}\left(\right.$ int $\left.X_{j}\right)$.

$(05)^{*} \exists 0<\gamma<1,0<\bar{\gamma}<\infty$ such that

$$
\sigma_{A, T_{A}}(n)=\sup \left\{\operatorname{diam} Y \mid Y \in \bigvee_{j=0}^{n-1} T_{A}^{-j}\left(Q_{A}\right)\right\} \leq \bar{\gamma} \gamma^{n}
$$

and there exists $\theta>0$ such that

$(06)^{*} \forall \underline{i}=\left(i_{1} \ldots i_{|\underline{\mid}|}\right) \in I_{A}$ and all $0 \leq j<|\underline{i}|, \exists 0<L_{\phi}\left(i_{j+1} \ldots i_{|\underline{\mid}|}\right)<\infty$ satisfying

$$
\left|\phi\left(v_{i_{j+1} \ldots i_{|\underline{i}|}}(x)\right)-\phi\left(v_{i_{j+1} \ldots i_{|\underline{\mid}|}}(y)\right)\right| \leq L_{\phi}\left(i_{j+1} \ldots i_{|\underline{i}|}\right) d(x, y)^{\theta} \quad(\forall x, y \in A)
$$


and

$$
\sup _{\underline{i} \in I_{A}} \sum_{j=0}^{|\underline{i}|-1} L_{\phi}\left(i_{j+1} \ldots i_{|\underline{i}|}\right)<\infty .
$$

Since the conditions $(05-06)^{*}$ allow us to establish the WBV property of $\phi_{A}^{(s)}$, by Theorem $1, \exists \lim _{n \rightarrow \infty} \frac{1}{n} \log Z_{n}\left(\phi_{A}^{(s)}\right):=P_{\text {top }}\left(T_{A}, \phi_{A}^{(s)}\right)$, where

$$
Z_{n}\left(\phi_{A}^{(s)}\right)=\sum_{\left(\underline{i}_{1} \ldots \underline{i}_{n}\right) \in I_{A}^{n}} \sum_{{\underline{\underline{i}_{1}} \ldots \underline{i}_{n} x=x}_{m=0}} \exp \left[\sum_{m=0}^{n-1} \phi_{A}^{(s)} T_{A}^{m}(x)\right] .
$$

Furthermore, (05-06)* guarantee equi-Hölder continuity of $\left\{\phi_{A}^{(s)} v_{\underline{i}}: \underline{i} \in I_{A}\right\}$ and $\sum_{n=1}^{\infty} \operatorname{Var}_{n}\left(T_{A}, \phi_{A}^{(s)}\right)<\infty$. Hence if $\left\|\mathcal{L}_{\phi_{A}^{(s)}} 1\right\|<\infty$, then $\mathcal{L}_{\phi_{A}^{(s)}}: C(A) \rightarrow C(A)$.

Theorem 7 (A construction of $\sigma$-finite conformal measures via induced maps). Let $(T, X, Q)$ be a transitive FRS Markov system and let $\phi \in \mathcal{W}(T)$. Suppose that there exists a full cylinder $X_{j} \in Q$ satisfying (05)* and (06)* for $A:=\operatorname{cl}\left(\right.$ int $\left.X_{j}\right)$. If

$$
\left\|\mathcal{L}_{\phi_{A}^{(0)}-R_{A} \min \left\{0, P_{\mathrm{top}}\left(T_{A}, \phi_{A}^{(0)}\right)\right\}} 1\right\|<\infty
$$

then

(i) $\exists s_{0} \in \mathbb{R}$ with $P_{\text {top }}\left(T_{A}, \phi_{A}^{\left(s_{0}\right)}\right)=0$ (a generalized Bowen's equation);

(ii) there exists a Borel probability measure $\nu_{A}$ on $(A, \mathcal{F} \cap A)$ with $\nu_{A}(\{x \in$ $\left.\left.A \mid R_{A}(x)<\infty\right\}\right)=1$ satisfying $\mathcal{L}_{\phi_{A}^{\left(s_{0}\right)}}^{*} \nu_{A}=\nu_{A} ;$

(iii) there exists a $\sigma$-finite measure $\nu$ on $X$ satisfying $\mathcal{L}_{\phi}^{*} \nu=\left[\exp s_{0}\right] \nu$ and $\nu\left(\bigcup_{i \in I} \partial X_{i}\right)=0$

(iv) in particular, if $\nu$ is finite, then $P_{\mathrm{top}}(T, \phi)=s_{0}$.

Proof of Theorem 7. By Lemmas 13-14 and Proposition 1, we have the existence of $s \in \mathbb{R}$ for which $P_{\text {top }}\left(T_{A}, \phi_{A}^{(s)}\right)=0$ and $\mathcal{L}_{\phi_{A}^{(s)}}^{*}$ admits an eigenvalue 1 . Then we can apply the main theorem in [5] so that (i)-(iv) are obtained.

The next result gives a criterion of finiteness of $\nu$.

Proposition 5 (A criterion of finiteness of $\nu$ ). Suppose that all assumptions in Theorem 7 are satisfied. Then $\nu(X)=\int_{A} \exp \left[s_{0}-\phi\right] d \nu_{A}+1$. In particular, if $\inf _{x \in A} \phi(x)>-\infty$, then $\nu$ is finite.

Proof of Proposition 5. Let $I^{\prime}=\left\{\underline{i} \mid X_{\underline{i}} \subset D_{|\underline{i}|}\right\}$ : First we note the following formula of $\nu$, which was obtained in [5]:

$$
\nu(E)=\sum_{\underline{i} \in I^{\prime}} \int_{A} 1_{E}\left(v_{\underline{i}} x\right) \exp \left[\sum_{l=0}^{|\underline{i}|-1} \phi T^{l}\left(v_{\underline{i}} x\right)-s_{0}|\underline{i}|\right] d \nu_{A}(x)+\int_{A} 1_{E}(x) d \nu_{A}(x) .
$$

Then we see that $\nu(X)$ is equal to

$$
\sum_{k=1}^{\infty} \sum_{\underline{j} \in I_{A}, \underline{j} \mid=k+1} \int_{T_{A} \underline{v}_{\underline{j}}(A)} \exp \left[\sum_{l=1}^{k}\left(\phi T^{l}-s_{0}\right)\left(v_{\underline{j}} x\right)\right] d \nu_{A}(x)+1
$$


because of the fact that $X_{\underline{i}} \subset T A\left(\forall \underline{i} \in I^{\prime}\right)$. By conformality of $\nu_{A}$ this coincides with

$$
\sum_{k=1}^{\infty} \sum_{\underline{j} \in I_{A},|\underline{j}|=k+1} \int_{v_{\underline{\underline{j}}}(A)} \exp \left[\left(\sum_{l=1}^{k} \phi T^{l}-s_{0}\right)(x)\right] \exp \left[-\phi_{A}^{\left(s_{0}\right)}(x)\right] d \nu_{A}(x)+1 .
$$

\section{§7. The CONSTRUCtion of MUtUally Singular EQUilibrium STATES}

In this section, we show the existence of mutually singular non-atomic equilibrium states by using induced systems.

Lemma 15. Let $(T, X, Q)$ be a transitive FRS Markov system and let $\phi \in \mathcal{W}(T)$. Suppose that there exists a sequence of full cylinders $\left\{X_{i}\right\}_{i=1}^{M}(M \leq \infty)$ that satisfies $(05)^{*}$ and $(06)^{*}, \inf _{x \in A_{i}} \phi(x)>-\infty$, and

$$
\left\|\mathcal{L}_{\phi_{A_{i}} \min \left\{0, P_{\mathrm{top}}\left(T_{A_{i}}, \phi_{A_{i}}^{(0)}\right)\right\}} 1\right\|<\infty
$$

for each $A_{i}=\operatorname{cl}\left(\right.$ int $\left.X_{i}\right)$. Let $\Gamma_{0}:=X$ and for each $i \geq 0$ define inductively $\Gamma_{i+1}=$ $\bigcap_{n=0}^{\infty} T^{-n}\left(\Gamma_{i} \cap A_{i+1}^{c}\right)\left(\subset \Gamma_{i}\right)$. We assume that for each $i \geq 0$,

$$
H_{\nu_{\Gamma_{i} \cap A_{i+1}}}\left(Q_{\Gamma_{i} \cap A_{i+1}}\right)<\infty, \quad \int_{\Gamma_{i} \cap A_{i+1}} R_{\Gamma_{i} \cap A_{i+1}} d \nu_{\Gamma_{i} \cap A_{i+1}}<\infty
$$

for the Borel probability measure $\nu_{\Gamma_{i} \cap A_{i+1}}$ on $\Gamma_{i} \cap A_{i+1}$ obtained in Theorem 7 . If $\bigcap_{i=1}^{M} \Gamma_{i}:=\Gamma$ consists of periodic points, then there exists a $T$-invariant ergodic probability measure $\mu$ equivalent to an $\exp \left[P_{\mathrm{top}}(T, \phi)-\phi\right]$-conformal measure $\nu$ that satisfies the following variational principle:

$P_{\text {top }}(T, \phi)=h_{\mu}(T)+\int_{X} \phi d \mu=\sup \left\{h_{m}(T)+\int_{X} \phi d m \mid m \in M_{T}(X, \phi)\right.$ is ergodic $\}$.

The equilibrium state $\mu$ for $\phi$ is not necessarily unique.

Theorem 8 (Phase transition and singular equilibrium states). We assume all conditions in Lemma 15. If $\Gamma:=\bigcap_{i=1}^{M} \Gamma_{i}$ consists of generalized indifferent periodic points with respect to $\phi$, then there exists a sequence of ergodic equilibrium states $\left\{\mu_{i}\right\}_{i=1}^{M}$ that are mutually singular and the set of equilibrium states for $\phi$ is the convex hull of $\left\{\mu_{i}\right\}_{i=1}^{M}$ and the set of invariant Borel probability measures supported on $\Gamma$.

Lemma 16 (Kac's formula). If $\int_{A} R_{A} d\left(\left.\nu\right|_{A}\right)<\infty$ and $\mu_{A}$ is a $T_{A}$-invariant ergodic probability measure equivalent to $\left.\nu\right|_{A}$, then the next formula gives a $T$-invariant ergodic probability measure $\mu$ equivalent to $\nu$ :

$$
\mu(E) / \mu(A)=\int_{A} \sum_{i=0}^{R_{A}(x)-1} 1_{E} \circ T^{i}(x) d \mu_{A}(x)(\forall E \in \mathcal{F}) .
$$

Lemma 17 (Finite entropy condition). Suppose that for $s_{i}:=P_{\text {top }}\left(\left.T\right|_{\Gamma_{i-1}}, \phi\right)$, $\int_{\Gamma_{i-1} \cap A_{i}} \phi_{\Gamma_{i-1} \cap A_{i}}^{\left(s_{i}\right)} d \nu_{\Gamma_{i-1} \cap A_{i}}>-\infty$. Then $H_{\nu_{\Gamma_{i} \cap A_{i+1}}}\left(Q_{\Gamma_{i} \cap A_{i+1}}\right)<\infty$. 
Proof of Lemma 17. Let $A=\Gamma_{i} \cap A_{i+1}$ and $s=s_{i}$. Since we have $\forall \underline{j} \in I_{A}$,

$$
\nu_{A}\left(X_{\underline{j}}\right)=\int_{A} \exp \left[\phi_{A}^{(s)}\left(v_{\underline{j}}(x)\right)\right] d \nu_{A}(x) \geq \exp [-s|\underline{j}|] \inf _{x \in v_{\underline{j}}(A)} \exp \left[\phi_{A}^{(0)}(x)\right],
$$

the bounded distortion for $\phi_{A}^{(0)}$ allows us to see that

$$
\begin{aligned}
\int_{A}\left(-\phi_{A}^{(0)}\right) d \nu_{A} & \geq-\log C-\sum_{\underline{j} \in I_{A}} \int_{X_{\underline{j}}} \log \left(\inf _{x \in v_{\underline{j}}(A)} \exp \left[\phi_{A}^{(0)}(x)\right]\right) d \nu_{A}(x) \\
& \geq-\log C+\sum_{\underline{j} \in I_{A}} \int_{X_{\underline{j}}}(-s|\underline{j}|) d \nu_{A}-\sum_{\underline{j} \in I_{A}} \nu_{A}\left(X_{\underline{j}}\right) \log \left(\nu_{A}\left(X_{\underline{j}}\right)\right),
\end{aligned}
$$

where $C$ is the bounded distortion constant $\exp \left[D \bar{\gamma}(1-\gamma)^{-1}(\operatorname{diam} X)^{\theta}\right]$. These inequalities allow one to establish

$$
H_{\nu_{A}}\left(Q_{A}\right) \leq \int_{A}\left(-\phi_{A}^{(0)}\right) d \nu_{A}+\log C+s \sum_{\underline{j} \in I_{A}} \int_{X_{\underline{j}}} R_{A} d \nu_{A}<\infty .
$$

Proof of Lemma 15. Since $\sum_{n=1}^{\infty} \operatorname{Var}_{n}\left(T_{A_{1}}, \phi_{A_{1}}^{(s)}\right)<\infty$ is satisfied, it follows from Theorem 7 that $\exists \nu_{A_{1}}$ on $A_{1}$ satisfying $\mathcal{L}_{\phi_{A_{1}}^{(s)}}^{*} \nu_{A_{1}}=\nu_{A_{1}}$ for $s=P_{\text {top }}(T, \phi)$ and $\exists \nu$ an $\exp \left[P_{\text {top }}(T, \phi)-\phi\right]$-conformal measure on $X$. Furthermore, by Proposition 5 we see that $\frac{\left.\nu\right|_{A_{1}}}{\nu\left(A_{1}\right)}=\nu_{A_{1}}$. The bounded distortion allows one to obtain an ergodic $T_{A_{1}}$ invariant probability measure $\mu_{A_{1}} \sim \nu_{A_{1}}$ with a density $d \mu_{A_{1}} / d \nu_{A_{1}}$ away from zero and infinity. Furthermore, by [14] there exists an equilibrium state $\mu_{A_{1}}$ for $\phi_{A_{1}}^{(s)}$ with respect to $T_{A_{1}}$ that is ergodic. In particular, since $H_{\nu_{A_{1}}}\left(Q_{A_{1}}\right)<\infty$ we have for $s_{1}=P_{\text {top }}(T, \phi)$,

$$
P_{\text {top }}\left(T_{A_{1}}, \phi_{A_{1}}^{\left(s_{1}\right)}\right)=0=h_{\mu_{A_{1}}}\left(T_{A_{1}}\right)+\int_{A_{1}} \phi_{A_{1}}^{\left(s_{1}\right)} d \mu_{A_{1}} \geq h_{m_{A_{1}}}\left(T_{A_{1}}\right)+\int_{A_{1}} \phi_{A_{1}}^{\left(s_{1}\right)} d m_{A_{1}}
$$

for all $T_{A_{1}}$ - invariant probability measures $m_{A_{1}} \in M_{T_{A_{1}}}\left(A_{1}, \phi_{A_{1}}^{\left(s_{1}\right)}\right)$ (cf. 14]). These inequalities and Lemma 16 allow us to have a $T$-invariant ergodic probability measure $\mu_{1} \sim \nu$ that satisfies $\mu_{1}\left(A_{1}\right)>0, \mu_{1}\left(\Gamma_{1}\right)=0$ and

$$
\begin{gathered}
0=\mu_{1}\left(A_{1}\right)^{-1}\left(h_{\mu_{1}}(T)+\int_{X}\left(\phi-s_{1}\right) d \mu_{1}\right) \\
\geq m\left(A_{1}\right)^{-1}\left(h_{m}(T)+\int_{X}\left(\phi-s_{1}\right) d m\right)
\end{gathered}
$$

for all $T$-invariant ergodic probability measures $m \in M_{T}(X, \phi)$ satisfying $m\left(A_{1}\right)>$ $0 .(* *)$ is equivalent to the inequalities : $s_{1}=h_{\mu_{1}}(T)+\int_{X} \phi d \mu_{1} \geq h_{m}(T)+\int_{X} \phi d m$. On the other hand, any $m \in M_{T}(X, \phi)$ satisfying $m\left(A_{1}\right)=0$ is supported on $\Gamma_{1}$. In fact, since $X=\left(\bigcup_{i=0}^{\infty} T^{-i} A_{1}\right) \cup\left(\bigcap_{i=0}^{\infty}\left(T^{-i} A_{1}\right)^{c}\right), m\left(A_{1}\right)=0$ and $T$-invariance of $m$ give $m\left(\Gamma_{1}\right)=1$. Thus the set of all $T$-invariant probability measures $m$ supported on $\Gamma_{1}$ coincides with the set of all $T$-invariant probability measures $m$ with $m\left(A_{1}\right)=0$. Since $\left(\left.T\right|_{\Gamma_{1}}, \Gamma_{1}, Q_{\Gamma_{1}}:=Q \cap \Gamma_{1}\right)$ is a subsystem of $(T, X, Q)$, we can apply the above arguments for the induced system $\left(T_{A_{2} \cap \Gamma_{1}}, A_{2} \cap \Gamma_{1}, Q_{A_{2} \cap \Gamma_{1}}\right)$. That is, for $s_{2}=P_{\mathrm{top}}\left(\left.T\right|_{\Gamma_{1}}, \phi\right):=\lim _{n \rightarrow \infty} \frac{1}{n} \log \sum_{\underline{i}:|\underline{i}|=n} \sum_{v_{\underline{i}} x=x, x \in \Gamma_{1}} \exp \left[\sum_{h=0}^{n-1} \phi T^{h}(x)\right]$ and for the associated potential $\phi_{A_{2} \cap \Gamma_{1}}^{\left(s_{2}\right)}(x)=\sum_{h=0}^{R_{A_{2} \cap \Gamma_{1}}(x)-1} \phi T^{h}(x)-s_{2} R_{A_{2} \cap \Gamma_{1}}(x)$ 
our assumptions allow us to establish the bounded distortion for $\phi_{A_{2} \cap \Gamma_{1}}^{\left(s_{2}\right)}$ so that there exists a $T_{A_{2} \cap \Gamma_{1}}$-invariant ergodic probability measure $\mu_{A_{2} \cap \Gamma_{1}}$ that satisfies

$$
\begin{aligned}
0 & =h_{\mu_{A_{2} \cap \Gamma_{1}}}\left(T_{A_{2} \cap \Gamma_{1}}\right)+\int_{A_{2} \cap \Gamma_{1}} \phi_{A_{2} \cap \Gamma_{1}}^{\left(s_{2}\right)} d \mu_{A_{2} \cap \Gamma_{1}} \\
& \geq h_{m_{A_{2} \cap \Gamma_{1}}}\left(T_{A_{2} \cap \Gamma_{1}}\right)+\int_{A_{2} \cap \Gamma_{1}} \phi_{A_{2} \cap \Gamma_{1}}^{\left(s_{2}\right)} d m_{A_{2} \cap \Gamma_{1}}
\end{aligned}
$$

for all $T_{A_{2} \cap \Gamma_{1}}$ - invariant ergodic probability measures $m_{A_{2} \cap \Gamma_{1}} \in M_{T_{A_{2} \cap \Gamma_{1}}}\left(A_{2} \cap \Gamma_{1}\right.$, $\phi_{A_{2} \cap \Gamma_{1}}^{\left(s_{2}\right)}$ ). Let $\mu_{2}$ be the $T$-invariant ergodic probability measure supported on $\Gamma_{1}$ arising from $\mu_{A_{2} \cap \Gamma_{1}}$ via Kac's formula. Then $s_{2}=h_{\mu_{2}}(T)+\int_{X} \phi d \mu_{2} \geq h_{m}(T)+$ $\int_{X} \phi d m$ for all $T$-invariant ergodic probability measures $m \in M_{T}(X, \phi)$ supported on $\Gamma_{1}$ that satisfy $m\left(\Gamma_{1} \cap A_{2}\right)>0$. Inductively we have a decreasing sequence $\left\{s_{i}\right\}_{i \in \mathbb{N}}$, where $s_{i}=P_{\text {top }}\left(\left.T\right|_{\Gamma_{i-1}}, \phi\right)$ and a sequence of $T$-invariant ergodic probability measures $\left\{\mu_{i}\right\}_{i=1}^{M}$ such that $\mu_{i}$ is supported on $\Gamma_{i-1}, \mu_{i}\left(\Gamma_{i-1} \cap A_{i}\right)>0$ and $s_{i}$ satisfies $s_{i}=h_{\mu_{i}}(T)+\int_{X} \phi d \mu_{i} \geq h_{m}(T)+\int_{X} \phi d m$ for all $T$-invariant ergodic probability measures $m \in M_{T}(X, \phi)$ supported on $\Gamma_{i-1}$ with $m\left(\Gamma_{i-1} \cap A_{i}\right)>0$. Since $\mu_{i}\left(\Gamma_{i-1}\right)=1$ and $\mu_{i}\left(\Gamma_{i}\right)=0,\left\{\mu_{i}\right\}_{i=1}^{M}$ are mutually singular. Finally, for every $T$-invariant measure supported on $\Gamma$ that consists of periodic points we have $s_{1} \geq h_{m}(T)+\int_{X} \phi d m$. Since $\left\{s_{i}\right\}_{i \in \mathbb{N}}$ is decreasing, we complete the proof.

\section{$\S 8$. Applications}

In this section, we show some examples of transitive FRS Markov systems to which our theorems 1-8 can apply.

Example 1 (Brun's map [13], [18], [20]). Let $X=\left\{\left(x_{1}, x_{2}\right) \in \mathbb{R}^{2}: 0 \leq x_{2} \leq x_{1} \leq\right.$ $1\}$, and let

$$
X_{i}=\left\{\left(x_{1}, x_{2}\right) \in X: x_{i}+x_{1} \geq 1 \geq x_{i+1}+x_{1}\right\}
$$

for $i=0,1,2$ where we put $x_{0}=1$ and $x_{3}=0 . T$ is defined by

$$
\begin{aligned}
& T\left(x_{1}, x_{2}\right)=\left(\frac{x_{1}}{1-x_{1}}, \frac{x_{2}}{1-x_{1}}\right) \text { on } X_{0}, \\
& T\left(x_{1}, x_{2}\right)=\left(\frac{1}{x_{1}}-1, \frac{x_{2}}{x_{1}}\right) \text { on } X_{1}, \\
& T\left(x_{1}, x_{2}\right)=\left(\frac{x_{2}}{x_{1}}, \frac{1}{x_{1}}-1\right) \text { on } X_{2} .
\end{aligned}
$$

Then $Q=\left\{X_{i}\right\}_{i=0}^{2}$ is a Bernoulli partition and $(0,0)$ is an indifferent fixed point (i.e., $|\operatorname{det} D T(0,0)|=1)$. Since $T$ is a continuous piecewise $C^{2}$ map and $\sigma(n)=$ $n^{-1}$, all conditions (01)-(04) are satisfied and dynamical instability is polynomial. We see that $\phi=-\log |\operatorname{det} D T|$ is piecewise Lipschitz continuous so that $\phi$ is a potential of WBV. Furthermore, since each periodic point is contained in a single cylinder the property (1) is satisfied. Define $B_{1}=X_{1} \cup X_{2}$. Then $T^{*}$ satisfies the uniformly expanding property and a direct calculation allows us to establish (06) for $\phi=-\log |\operatorname{det} D T|$ (see [18] for more details). Hence we can apply Theorems 1-8. In particular, we can see that $P_{\text {top }}(T, \phi)=P_{\text {top }}\left(T^{*}, \phi^{*}\right)=0$.

Example 2 (Inhomogeneous Diophantine approximations [13], 15], 16], [17, [19], [20], [21]). For the transformation defined in the introduction (Example A), we can directly verify all conditions (01)-(04). In fact, we can introduce an index set

$$
I=\left\{\left(\begin{array}{l}
a \\
b
\end{array}\right): a, b \in \mathbb{Z}, a>b>0, \text { or } a<b<0\right\}
$$

and a partition $\left\{X_{\left(\begin{array}{c}a \\ b\end{array}\right)}:\left(\begin{array}{c}a \\ b\end{array}\right) \in I\right\}$, where $X_{\left(\begin{array}{c}a \\ b\end{array}\right)}=\left\{(x, y) \in X: a=\left[\frac{1-y}{x}\right]-\right.$ $\left.\left[-\frac{y}{x}\right], b=-\left[-\frac{y}{x}\right]\right\}$. Although $\phi=-\log |\operatorname{det} D T|$ fails (piecewise) Hölder continuity, 
we can verify $V_{n}(\phi) \leq \log (1+\sigma(n-2))$ and $\sigma(n)=O\left(n^{-1}\right)$ (see [15, 20]) (cf. [16], [19], [21]). Hence $\phi=-\log |\operatorname{det} D T|$ is a potential of WBV. Since each periodic point is contained in a single cylinder, the property (1) in Lemma 6 is satisfied. Let $D_{n}$ be the union of cylinders of rank $n$ containing indifferent periodic points and let $B_{n}=D_{n-1} \backslash D_{n}$. Then the jump transformation $T^{*}: \bigcup_{i=1}^{\infty} B_{i} \rightarrow X$ defined by $T^{*}(x, y)=T^{i}(x, y)$ for $(x, y) \in B_{i}$ satisfies exponential decay of diameter of cylinders (see [19]), and we can verify the validity of (06) for $\phi=-\log |\operatorname{det} D T|$. Indeed, for $\underline{i} \in I^{*}$ with $|\underline{i}|=n, L_{\phi}(\underline{i}) \leq 3 / n^{2}$ and so

$$
\sup _{\underline{i} \in I^{*}} \sum_{j=0}^{|\underline{i}|-1} L_{\phi}\left(i_{j+1} \ldots i_{|\underline{\mid}|}\right) \leq \sum_{n=1}^{\infty} \frac{3}{n^{2}}<\infty .
$$

Hence we have summability of $\operatorname{Var}_{n}\left(T^{*}, \phi^{*}\right)$, which allows us to apply Theorems $1-8$.

Example 3 (A complex continued fraction [5], 12, [15, 22]). For the transformation $T$, defined in the introduction (Example B), we define $X_{n \alpha+m \bar{\alpha}}=\{z \in X$ : $\left.[1 / z]_{1}=n \alpha+m \bar{\alpha}\right\}$ for each $n \alpha+m \bar{\alpha} \in I:=\left\{m \alpha+n \bar{\alpha}:(m, n) \in \mathbb{Z}^{2}-(0,0)\right\}$. Then we have a countable partition $Q=\left\{X_{a}\right\}_{a \in I}$ of $X$ that is a topologically mixing Markov partition and satisfies (01)-(03). The inverse branches to $T$ take the form $v_{j}(z)=1 /(j+z)$, where $j \in I$ and the $v_{j}$ satisfy (04). Therefore the inverse branches of the $n$th iterate of the transformation $T^{n}$ take the form

$$
v_{j_{1}, \ldots, j_{n}}(z)=\frac{p_{n}+z p_{n-1}}{q_{n}+z q_{n-1}} \text { and }\left|v_{j_{1}, \ldots, j_{n}}^{\prime}(z)\right|=\frac{1}{\left|q_{n}+z q_{n-1}\right|^{2}}
$$

where $p_{n}=j_{n} p_{n-1}+p_{n-2}$ and $q_{n}=j_{n} q_{n-1}+q_{n-2}, n \geq 1$, and $p_{-1}=\alpha, p_{0}=$ $0=q_{-1}$ and $q_{0}=\alpha$. If the string $j_{1}, \ldots, j_{n-1}$ corresponds to a cylinder that contains one of the indifferent points, but the longer string $j_{1}, \ldots, j_{n}$ corresponds to a sub-cylinder disjoint from the indifferent periodic points, then $v_{j_{1}, \ldots, j_{n}}$ is an inverse branch of the jump transformation $T^{*}$ which is uniformly expanding. For $\phi(z)=-\log \left|T^{\prime}(z)\right|$, WBV and (06) are satisfied. Further details can be found in 22] in which multifractal formalism was established by applying our Theorems 1-8.

\section{§9. Appendix - Proofs of Results in $\S \S 2$ and 3}

For the proof of Theorem 1, we first verify the following facts.

Lemma 18. (18-1) $\forall U_{k} \in \mathcal{U}, Z_{n}\left(U_{k}, \phi\right)>0$ for all $n>S$.

(18-2) $\forall U_{k} \in \mathcal{U}, \forall n, m>S, \underline{Z}_{n+m}\left(U_{k}, \phi\right) \geq \underline{Z}_{n}\left(U_{k}, \phi\right) \underline{Z}_{m}\left(U_{k}, \phi\right)$. (Subadditivity)

(18-3) $\forall U_{k} . U_{l} \in \mathcal{U}$,

$$
\begin{aligned}
& \underline{Z}_{n+s_{l}+s_{k}}\left(U_{l}, \phi\right) \geq \underline{Z}_{n}\left(U_{k}, \phi\right)\left(C_{s_{l}} C_{s_{k}}\right)^{-1} \\
& \quad \times \sup _{x \in X^{(l, k)}\left(s_{k}\right)} \exp \left[\sum_{h=0}^{s_{k}-1} \phi T^{h}(x)\right] \sup _{x \in X^{(k, l)}\left(s_{l}\right)} \exp \left[\sum_{h=0}^{s_{l}-1} \phi T^{h}(x)\right] .
\end{aligned}
$$

Proof of Lemma 18. (18-1) follows from Lemma 1 and Remark (A). (18-2) follows from the definition of $\underline{Z}_{n}(U, \phi)$ directly. Since $\underline{Z}_{n+s_{l}+s_{k}}\left(U_{l}, \phi\right)$ is bounded from below by

$$
\sum_{\underline{i}:|\underline{i}|=s_{k}, \operatorname{int}\left(T X_{i_{s_{k}}}\right)=U_{k}, \text { int } X_{i_{1}} \subset U_{l}} \inf _{x \in X_{\underline{i}}} \exp \left[\sum_{h=0}^{s_{k}-1} \phi T^{h}(x)\right]
$$




$$
\begin{aligned}
& \times \sum_{\underline{j}:|\underline{j}|=n, \operatorname{int}\left(T X_{j_{n}}\right)=U_{k} \supset \operatorname{int} X_{j_{1}}} \inf _{x \in X_{\underline{j}}} \exp \left[\sum_{h=0}^{n-1} \phi T^{h}(x)\right] \\
& \times \sum_{\underline{t}:|\underline{\mid}|=s_{l}, \text { int }\left(T X_{t_{s_{l}}}\right)=U_{l}, \text { int } X_{t_{1}} \subset U_{k}} \inf _{x \in X_{\underline{\underline{t}}}} \exp \left[\sum_{h=0}^{s_{l}-1} \phi T^{h}(x)\right]
\end{aligned}
$$

and the transitivity condition allows one to establish

$$
\sum_{\underline{i}:|\underline{i}|=s_{k}, \operatorname{int}\left(T X_{i_{s_{k}}}\right)=U_{k}, \operatorname{int} X_{i_{1}} \subset U_{l}} \inf _{x \in X_{\underline{i}}} \exp \left[\sum_{h=0}^{s_{k}-1} \phi T^{h}(x)\right] \geq \inf _{x \in X^{(l, k)}\left(s_{k}\right)} \exp \left[\sum_{h=0}^{s_{k}-1} \phi T^{h}(x)\right]
$$

and

$$
\sum_{\underline{t}:|\underline{t}|=s_{l}, i n t\left(T X_{s_{s_{l}}}\right)=U_{l}, \text { int } X_{t_{1}} \subset U_{k}} \inf _{x \in X_{\underline{\underline{t}}}} \exp \left[\sum_{h=0}^{s_{l}-1} \phi T^{h}(x)\right] \geq \inf _{x \in X^{(k, l)}\left(s_{l}\right)} \exp \left[\sum_{h=0}^{s_{l}-1} \phi T^{h}(x)\right],
$$

(18-3) follows from the WBV property of $\phi$.

Proof of Theorem 1. By (18-2) in Lemma 18 and the WBV property of $\phi$, both $\lim _{n \rightarrow \infty} \frac{1}{n} \log \bar{Z}_{n}(U, \phi)$ and $\lim _{n \rightarrow \infty} \frac{1}{n} \log \underline{Z}_{n}(U, \phi)$ exist for each $U \in \mathcal{U}$. Since $\underline{Z}_{n}(U, \phi) \leq Z_{n}(U, \phi) \leq \bar{Z}_{n}(U, \phi)$, by the WBV property of $\phi, \lim _{n \rightarrow \infty} \frac{1}{n} \log Z_{n}(U, \phi)$ also exists and all the limits coincide. By (18-3) in Lemma 18, it is obvious that the limit does not depend on $U$. Noting $\min _{1 \leq j \leq N} Z_{n}\left(\phi, U_{j}\right) \leq Z_{n}(\phi) \leq \sum_{j=1}^{N} Z_{n}\left(\phi, U_{j}\right)$ allows one to complete the proof.

In order to prove Theorem 3 we first show the next result.

Lemma 19. (19-1) $\forall V \in \mathcal{V}$ and $\forall x \in V, \mathcal{L}_{\phi}^{n} 1_{V}(x) \leq \sum_{U_{l} \supseteq V} \overline{Z_{n}}\left(U_{l}, \phi\right)$.

(19-2) $\forall x \in U_{k} \in \mathcal{U}, \mathcal{L}_{\phi}^{n} 1_{U_{k}}(x) \geq \underline{Z_{n}}\left(U_{k}, \phi\right)$.

(19-3) $Z_{n}(\phi) \geq C_{1}^{-1} C_{n-1}^{-1}\left\{\min _{1 \leq k \leq N} \sup _{y \in X^{(k)}(1)} \exp \phi(y)\right\}\left\|\mathcal{L}_{\phi}^{n-2} 1\right\|$, where $X^{(k)}(1) \in Q$ satisfies $X^{(k)}(1) \subset U_{k}$ and $T\left(\operatorname{int} X^{k}(1)\right)=\operatorname{int} X$.

Proof of Lemma 19. We first note that $V=\bigcup_{X_{j} \subset V} X_{j}$ because of the Markov property of $Q$. Then for $x \in V$ the following inequalities allow us to have the assertion in (19-1):

$$
\begin{aligned}
\mathcal{L}_{\phi}^{n} 1_{V}(x) & =\sum_{j \in I: X_{j} \subset V} \mathcal{L}_{\phi}^{n} 1_{X_{j}}(x)=\sum_{j \in I: X_{j} \subset V} \sum_{\left(j i_{2} \ldots i_{n}\right): x \in T X_{i_{n}}} \exp \left[\sum_{h=0}^{n-1} \phi T^{h}\left(v_{j i_{2} \ldots i_{n}} x\right)\right] \\
& =\sum_{j \in I: X_{j} \subset V} \sum_{U_{l} \in \mathcal{U}: x \in U_{l}} \sum_{\left(j i_{2} \ldots i_{n}\right): T X_{i_{n}}=U_{l}} \exp \left[\sum_{h=0}^{n-1} \phi T^{h}\left(v_{j i_{2} \ldots i_{n}} x\right)\right] \\
& =\sum_{U_{l} \in \mathcal{U}: U_{l} \supset V} \sum_{\left(j i_{2} \ldots i_{n}\right): T X_{i_{n}}=U_{l} \supset V \supset X_{j}} \exp \left[\sum_{h=0}^{n-1} \phi T^{h}\left(v_{j i_{2} \ldots i_{n}} x\right)\right] \\
& \leq \sum_{U_{l} \in \mathcal{U}: U_{l} \supset V} \exp \left[\sum_{h=0}^{n-1} \phi T^{h}\left(v_{j i_{2} \ldots i_{n}} x\right)\right] \\
& \leq \sum_{U_{l} \in \mathcal{U}: U_{l} \supset V} \bar{Z}_{n}\left(U_{l}, \phi\right) .
\end{aligned}
$$


For $x \in U_{k}$, we have the following inequalities, which give (19-2):

$$
\begin{aligned}
\mathcal{L}_{\phi}^{n} 1_{U_{k}}(x) & =\sum_{j \in I: X_{j} \subset U_{k}} \sum_{\left(j i_{2} \ldots i_{n}\right): x \in T X_{i_{n}}} \exp \left[\sum_{h=0}^{n-1} \phi T^{h}\left(v_{j i_{2} \ldots i_{n}} x\right)\right] \\
& =\sum_{j \in I: X_{j} \subset U_{k}} \sum_{U_{l} \in \mathcal{U}: x \in U_{l}} \sum_{\left(j i_{2} \ldots i_{n}\right): T X_{i_{n}}=U_{l}} \exp \left[\sum_{h=0}^{n-1} \phi T^{h}\left(v_{j i_{2} \ldots i_{n}} x\right)\right] \\
& \geq \sum_{\left(j i_{2} \ldots i_{n}\right): T X_{i_{n}}=U_{k} \supset X_{j}} \exp \left[\sum_{h=0}^{n-1} \phi T^{h}\left(v_{j i_{2} \ldots i_{n}} x\right)\right] \geq \underline{Z}_{n}\left(U_{k}, \phi\right) .
\end{aligned}
$$

By the WBV property of $\phi, Z_{n}(\phi)$ is bounded from below by

$$
C_{1}^{-1} C_{n-1}^{-1} \sum_{l=1}^{N} \sum_{\substack{i \in|i| i \mid=n, \operatorname{int}\left(T X_{i_{n}}\right)=U_{l} \supset \operatorname{int} X_{i_{1}}}} \sup _{x \in X_{i_{1}}} \exp [\phi(x)] \sup _{x \in T X_{i_{n}}} \exp \left[\sum_{h=0}^{n-2} \phi T^{h} v_{i_{2} \ldots i_{n}}(x)\right] .
$$

Then (19-3) follows from the Markov property and the strong transitivity.

Proof of Theorem 3. By (19-1,2) in Lemma 19, we have for $x \in V \subset U_{k}$,

$$
\limsup _{n \rightarrow \infty} \frac{1}{n} \log \mathcal{L}_{\phi}^{n} 1_{V}(x) \leq \lim _{n \rightarrow \infty} \frac{1}{n} \log N\left(\max _{1 \leq l \leq N} \bar{Z}_{n}\left(U_{l}, \phi\right)\right)=P_{\text {top }}(T, \phi)
$$

and $\liminf \operatorname{in}_{n \rightarrow \infty} \frac{1}{n} \log \mathcal{L}_{\phi}^{n} 1_{U_{k}}(x) \geq P_{\text {top }}(T, \phi)$. Our assumption $U_{k} \in \mathcal{V}$ implies $U_{k}=$ $V$. Hence we have the first assertion. The rest of the assertions follow from (19-3) in Lemma 19 immediately.

In order to prove Proposition 1, we need two sublemmas.

Sublemma A (Lemma 3.1 in [17]). $\bigcap_{n>0} D_{n}$ and $X^{*}$ are positively $T$-invariant. Furthermore, $\bigcup_{m=1}^{\infty} T^{*-m}\left(\bigcap_{n \geq 0} D_{n}\right)$ contains no periodic points.

Proof. The result follows from the equality (4) : $R(T x)=R(x)-1(R(x) \geq 2)$.

Sublemma B. Define

$$
\begin{gathered}
\mathcal{P}_{n}(X, T):=\left\{x \in X \mid \exists\left(i_{1} \ldots i_{n}\right) \in I^{n} \text { such that } v_{i_{1} \ldots i_{n}} x=x\right\} ; \\
\mathcal{P}_{n}\left(X^{*}, T^{*}\right):=\left\{x \in X \mid \exists\left(\underline{i}_{1} \ldots \underline{i}_{n}\right) \in I^{* n} \text { such that } v_{\underline{i}_{1} \ldots \underline{i}_{n}} x=x\right\} .
\end{gathered}
$$

Then $\forall x \in \mathcal{P}_{n}(X, T) \cap X^{*}, \exists 0<l \leq n, \exists y \in \mathcal{P}_{l}\left(X^{*}, T^{*}\right)$ such that $T^{j} y=x$ for some $j<R(y)$ and $\sum_{m=0}^{l-1} R\left(T^{* m}(y)\right)=n$. Furthermore, $\sum_{m=0}^{l-1}\left(\phi^{*}-s R\right) T^{* m}(y)=$ $\sum_{h=0}^{n-1}(\phi-s) T^{h}(x)$. Conversely, $\forall y \in \mathcal{P}_{l}\left(X^{*}, T^{*}\right)$ and $\forall 0 \leq j<R(y), T^{j} y \in$ $\mathcal{P}_{n}(X, T) \cap X^{*}$ and $\sum_{m=0}^{l-1} R\left(T^{* m}(y)\right)=n$.

Proof. Since $x \in \mathcal{P}_{n}(X, T) \cap X^{*}$ visits $B_{1}$ infinitely often, we can find a point $y \in \mathcal{P}_{l}\left(X^{*}, T^{*}\right)$ for some $l \leq n$ such that $T^{j} y=x$ for some $j<R(y)$ and 
$\sum_{m=0}^{l-1} R\left(T^{* m}(y)\right)=n$. By the property (4) the converse is also true. Since

$$
\begin{aligned}
\sum_{m=0}^{l-1}\left(\phi^{*}-s R\right) T^{* m}(y) & =\sum_{m=0}^{l-1} \sum_{h=0}^{R\left(T^{* m} y\right)-1} \phi T^{h}\left(T^{* m} y\right)-s n \\
& =\sum_{h=0}^{\sum_{m=0}^{l-1} R\left(T^{* m} y\right)-1}(\phi-s) T^{h}(y)
\end{aligned}
$$

we have the rest of the assertion.

Proof of Proposition 1. By Sublemma A we first note that $\mathcal{P}_{n}(X, T)=\left\{\mathcal{P}_{n}(X, T) \cap\right.$ $\left.\bigcap_{n \geq 0} D_{n}\right\} \cup\left\{\mathcal{P}_{n}(X, T) \cap X^{*}\right\}$. Then we see that $\zeta_{T, \phi}(\exp (-s))$ is equal to

$$
\zeta_{\left.T\right|_{\cap_{n}>0} D_{n}, \phi}(\exp (-s)) \exp \left[\sum_{n=1}^{\infty} \frac{\exp [-n s]}{n} \times \sum_{x \in \mathcal{P}_{n}(X, T) \cap X^{*}} \exp \left[\sum_{h=0}^{n-1} \phi T^{h}(x)\right]\right] .
$$

Define for $n \geq l>0 E_{n, l}^{*}:=\left\{y \in \mathcal{P}_{l}\left(X^{*}, T^{*}\right): \sum_{m=0}^{l-1} R\left(T^{* m}(y)\right)=n\right\}$ and

$$
\begin{gathered}
E_{n, l}:=\left\{x \in X^{*} \cap \mathcal{P}_{n}(X, T): \exists y \in X^{*} \cap \mathcal{P}_{l}\left(X^{*}, T^{*}\right) \text { and } \exists j<R(y)\right. \\
\text { such that } \left.\sum_{m=0}^{l-1} R\left(T^{* m}(y)\right)=n \text { and } x=T^{j} y\right\} .
\end{gathered}
$$

Then $\mathcal{P}_{l}\left(X^{*}, T^{*}\right)=\bigcup_{n \geq l} E_{n, l}^{*}$ and $\mathcal{P}_{n}(X, T) \cap X^{*}=\bigcup_{l \leq n} E_{n, l}$. By Sublemma B we see that for $x \in X^{*}$,

$$
\begin{aligned}
& \sum_{n=1}^{\infty} \frac{1}{n} \sum_{x \in \mathcal{P}_{n}(X, T) \cap X^{*}} \exp \left[\sum_{h=0}^{n-1}(\phi-s) T^{h}(x)\right] \\
& =\sum_{n=1}^{\infty} \sum_{l \leq n} \sum_{\left\{x, T x, \ldots T^{n-1} x\right\} \subset E_{n, l}} \exp \left[\sum_{h=0}^{n-1}(\phi-s) T^{h}(x)\right] \\
& =\sum_{n=1}^{\infty} \sum_{l \leq n} \sum_{\left\{y, T^{*} y, \ldots T^{* l-1} y\right\} \subset E_{n, l}^{*}} \exp \left[\sum_{m=0}^{l-1}\left(\phi^{*}-s R\right) T^{* m}(y)\right]
\end{aligned}
$$

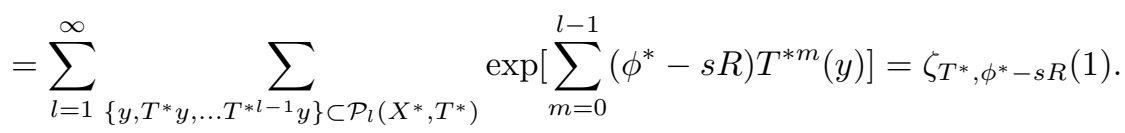

We complete the proof.

Proof of Lemma 7. (i) By Lemmas 13-14, we have continuity of the function $P_{\text {top }}\left(T^{*}, \phi^{*}-s R\right)$ on $\operatorname{int}\left\{s \in \mathbb{R} \mid P_{\text {top }}\left(T^{*}, \phi^{*}\right) \in \mathbb{R}\right\}$. Then the existence of a zero $s_{0} \geq 0$ of (GBE) follows from the standard argument. Since $P_{\text {top }}\left(T^{*}, \phi^{*}-s_{0} R\right)=0$, by Corollary 1 we have $s_{0} \leq P_{\text {top }}(T, \phi)$. If $P_{\text {top }}(T, \phi)<0$, then we have a contradiction. For (ii), replacing $\phi$ by $\phi-P_{\text {top }}\left(T^{*}, \phi^{*}\right)$ allows us to reduce to the case (i). (iii) Since the case $0 \leq P_{\text {top }}\left(T^{*}, \phi^{*}\right)<\infty$ is covered by Lemma $7(\mathrm{i})$, we suppose either $P_{\text {top }}\left(T^{*}, \phi^{*}\right)=\infty$ or $P_{\text {top }}\left(T^{*}, \phi^{*}\right)<0$. If $P_{\text {top }}\left(T^{*}, \phi^{*}\right)=\infty$, then $\sup \left\{s \in \mathbb{R}: P_{\text {top }}\left(T^{*}, \phi^{*}-s R\right)=\infty\right\}=0$. Hence $\forall s>0, P_{\text {top }}\left(T^{*}, \phi^{*}-s R\right)<\infty$. Since the function $s \rightarrow P_{\text {top }}\left(T^{*}, \phi^{*}-s R\right)$ is decreasing and continuous on $\operatorname{int}\{s \in$ $\left.\mathbb{R} \mid P_{\text {top }}\left(T^{*}, \phi^{*}-s R\right) \in \mathbb{R}\right\}$, we have $\lim _{s \rightarrow 0} P_{\text {top }}\left(T^{*}, \phi^{*}-s R\right)=\infty$ so that for sufficientlly small $s>0, P_{\text {top }}\left(T^{*}, \phi^{*}-s R\right)>0$. On the other hand, it follows from 
Corollary 1 that $P_{\text {top }}\left(T^{*}, \phi^{*}-s R\right) \leq 0$ for $s>P_{\text {top }}(T, \phi)$. Hence we have a zero $s_{0} \geq 0$ of $(\mathrm{GBE})$. If $P_{\text {top }}\left(T^{*}, \phi^{*}\right)<0$, then $\sup \left\{s \in \mathbb{R}: P_{\text {top }}\left(T^{*}, \phi^{*}-s R\right)=\infty\right\}=$ $P_{\text {top }}\left(T^{*}, \phi^{*}\right)$. The same argument as those for the previous case allows us to have a zero $s_{0} \geq P_{\text {top }}\left(T^{*}, \phi^{*}\right)$ of (GBE).

Proof of Theorem 4. By Lemma 8 we have for $\underline{i} \in I^{*}, \frac{d\left(\nu v_{\underline{i}}\right)}{d \nu}=\exp \left[\phi^{*}-s_{0} R\right] v_{\underline{i}}$. If $\underline{i}=\underline{i}_{1} \underline{i}_{2} \ldots \underline{i}_{n}$, then $\frac{d\left(\nu v_{i}\right)}{d \nu}(x)=\exp \left[\sum_{h=0}^{n-1} \phi T^{h} v_{\underline{i}_{1} \underline{i}_{2} \ldots \underline{i}_{n}} x-s_{0} n\right]$. Since the property (4) : $R(T x)=R(x)-1(R(x) \geq 2)$ implies $\underline{i}_{2} \underline{i}_{3} \cdots \underline{i}_{n} \in I^{*}$, the equality

$$
\frac{d\left(\nu \underline{v}_{\underline{i}}\right)}{d \nu}=\frac{d\left(\nu{\underline{i_{1}}}_{1}\right)}{d \nu}\left(\underline{i}_{2} \underline{i}_{3} \ldots \underline{i}_{n} x\right) \frac{d\left(\nu{\underline{v_{2}}}_{\underline{i}_{3}} \ldots \underline{\underline{i}}_{n}\right)}{d \nu}(x)
$$

allows one to see that $\forall X_{i} \subset D_{1}, \frac{d\left(\nu v_{i}\right)}{d \nu}(x)=\exp \left[\phi v_{i}(x)-s_{0}\right]\left(\forall x \in X^{*}\right)$. On the other hand, we know that the above equality holds for $X_{i} \subset B_{1}$ since $i \in$ $I^{*}$. Finally, we establish $\forall X_{i} \in Q,\left.\frac{d(\nu T)}{d \nu}\right|_{X_{i}}(x)=\exp \left[s_{0}-\phi(x)\right]\left(\forall x \in X^{*}\right)$. It follows from Lemma 2 and Theorem 2.1 in [18] that $s_{0}=P_{\text {top }}(T, \phi)$. The assertion $\nu\left(\bigcup_{i \in I} \partial X_{i}\right)\left(=\nu\left(\bigcup_{i \in I} \partial v_{i}(X)\right)\right)=0$ follows from $\nu($ int $X)=1$, which is obtained by Lemma 8 . We complete the proof.

\section{REFERENCES}

1. J. Aaronson, M. Denker and M. Urbański, Ergodic theory for Markov fibred systems and parabolic rational maps, Trans. Amer. Math. Soc. 337 (1993), 495-548. MR 94g:58116

2. R. Bowen, Equilibrium states and the ergodic theory of Anosov diffeomorphisms, Springer Lecture Notes in Mathematics 470, Springer, 1975. MR 56:1364

3. M. Denker, F. Przytycki and M. Urbański, On the transfer operator for rational functions on the Riemann sphere, Ergodic Theory and Dynamical Systems 16 (1996), 255-266. MR 97e:58197

4. M. Denker and M. Urbański, On the existence of conformal measures, Trans. Amer. Math. Soc. 328 (1991), 563-587. MR 92k:58155

5. M. Denker and M. Yuri, A note on the construction of nonsingular Gibbs measures, Colloquium Mathematicum 84/85 (2000), 377-383. MR 2001k:37013

6. M. Denker and M. Yuri, Partially defined infinite iterated functional systems, In preparation.

7. D. Fiebig, U. Fiebig and M. Yuri, Pressures and Equilibrium states for countable Markov shifts, To appear in Israel J. Math.

8. P. H. Hanus, R. D. Mauldin and M. Urbański, Thermodynamic formalism and multi-fractal analysis of conformal infinite iterated functional systems, Acta Math. Hungar. 96 (2002), 27-98.

9. R. D. Mauldin and M. Urbański, Parabolic iterated function systems, Ergodic Theory and Dynamical Systems 20 (2000), 1423-1447. MR 2001m:37047

10. Omri Sarig, Thermodynamic formalism for countable Markov shifts., Ergodic Theory and Dynamical Systems 19 (1999), 1565-1593. MR 2000m:37009

11. Y. B. Pesin, Dimension Theory in Dynamical Systems. Contemporary Views and Applications, Chicago University Press, 1997. MR 99b:58003

12. M. Pollicott and M. Yuri, Zeta functions for certain multi-dimensional nonhyperbolic maps, Nonlinearity 14 (2001), 1265-1278. MR 2002h:37036

13. F. Schweiger, Ergodic theory of fibred systems and metric number theory, Oxford University Press, Oxford, 1995. MR 97h:11083

14. P. Walters, Invariant measures and equilibrium states for some mappings which expand distances, Trans. Amer. Math. Soc. 236 (1978), 121-153. MR 57:6371

15. M. Yuri, On a Bernoulli property for multi-dimensional mappings with finite range structure, Tokyo J. Math 9 (1986), 457-485. MR 88d:28023

16. M. Yuri, On the convergence to equilibrium states for certain nonhyperbolic systems, Ergodic Theory and Dynamical Systems 17 (1997), 977-1000. MR 98f:58155

17. M. Yuri, Zeta functions for certain nonhyperbolic systems and topological Markov approximations, Ergodic Theory and Dynamical Systems 18 (1998), 1589-1612. MR 2000j:37024 
18. M. Yuri, Thermodynamic formalism for certain nonhyperbolic maps, Ergodic Theory and Dynamical Systems 19 (1999), 1365-1378. MR 2001a:37012

19. M. Yuri, Statistical properties for nonhyperbolic maps with finite range structure, Trans. Amer. Math. Soc. 352 (2000), 2369-2388. MR 2000j:37009

20. M. Yuri, Weak Gibbs measures for certain nonhyperbolic systems, Ergodic Theory and Dynamical Systems 20 (2000), 1495-1518. MR 2002d:37011

21. M. Yuri, On the speed of convergence to equilibrium states for multi-dimensional maps with indifferent periodic points., Nonlinearity 15 (2002), 429-445. MR 2002k:37006

22. M. Yuri, Multifractal Analysis of weak Gibbs measures for Intermittent Systems., Commun. Math. Phys. 230 (2002), 365-388.

Department of Business Administration, Sapporo University, Nishioka, Toyohira-ku, SAPPORO 062-8520, JAPAN

E-mail address: yuri@math.sci.hokudai.ac.jp, yuri@mail-ext.sapporo-u.ac.jp 